\title{
THE CIRCUMSTELLAR ENVIRONMENT OF IRAS 05338-0624
}

\author{
Joseph P. McMullin, ${ }^{1}$ Lee G. Mundy, ${ }^{1}$ and Geoff A. Blake ${ }^{2}$ \\ Received 1994 March 25 ; accepted 1994 June 16
}

\begin{abstract}
Millimeter continuum and spectral line observations with $10^{\prime \prime}, 30^{\prime \prime}$, and $60^{\prime \prime}$ resolution are used to characterize the structure and chemistry of the gas around the young, embedded star, IRAS 05338-0624. On arcminute scales, emission from dense gas tracers outline an isolated condensation centered on the IRAS source position. The condensation is characterized by a size of $\sim 60^{\prime \prime}$, a density of $2 \times 10^{5} \mathrm{~cm}^{-3}$, and a virial mass of $40 M_{\odot}$. Interferometric CS $J=2-1$ observations show two peaks, one toward the continuum peak and the other toward a position 14" west and $8^{\prime \prime}$ south. Single-dish maps of $\mathrm{SO}, \mathrm{CH}_{3} \mathrm{OH}$, and $\mathrm{SiO}$ show pronounced wing emission to the west of the IRAS source, which interferometer observations reveal to be a compact region of outflow activity. CS emission at redshifted and blueshifted velocities reveals a bipolar outflow oriented with a position angle of $45^{\circ}$, while $\mathrm{SiO}$ emission appears to be tracing a fast shock interaction region at the CS red-lobe peak, $14^{\prime \prime}$ west and $8^{\prime \prime}$ south of the $I R A S$ source. Finally, $\mathrm{H}^{13} \mathrm{CO}^{+}$emission traces clumps of quiescent gas toward the IRAS source and adjacent to the blue lobe of the outflow.

Column densities and molecular fractional abundances are derived to explore the interaction between the surrounding condensation and the young stellar object. We find evidence for gas phase depletions within the overall condensation in several gas tracers $(\mathrm{CO}, \mathrm{CS}, \mathrm{HCN}, \mathrm{SO})$ but not in the region immediately around the young stellar object. Enhanced abundances of $\mathrm{SO}, \mathrm{CH}_{3} \mathrm{OH}$, and $\mathrm{SiO}$ (by factors of $4,>100,>1000$, respectively) are observed in the shocked gas; these enhancements may be explained in terms of a nondissociative shock liberating mantle materials that contain some amount of refractory materials, a moderate velocity dissociative shock in which only minor sputtering of Si occurs, or a shock that impacts surrounding material with a range of speeds.

Subject headings : circumstellar matter - ISM : jets and outflows - radio lines: stars stars: individual (IRAS 05338-0624) - stars: pre-main-sequence
\end{abstract}

\section{INTRODUCTION}

It is now clear that infall and outflow motions are characteristic of star formation (Shu et al. 1993), raising the possibility that both accretionary and shock processes modify the chemical composition of gas surrounding young stellar objects (YSOs). Although outflow activity is readily identified in starforming environments (e.g., Lada 1985), infall activity and the corresponding chemical chemical changes wrought by infall such as depletions of gas phase molecules (e.g., Rawlings et al. 1992) have been far more difficult to prove. Despite this, depleted abundances have been suggested for a number of starforming regions (e.g., Mauersberger et al. 1992; Blake, van Dishoeck, \& Sargent 1992; Goldsmith et al. 1992). Additionally, measurements of the physical and infrared properties of ices have confirmed the vital interaction between the gaseous and solid phases, and the proclivity for gas phase depletions to occur in dense clouds (e.g., Sandford \& Allamandola 1993). During the early stages of stellar genesis, depletion of gas phase tracer molecules onto grain mantles can occur rapidly at high densities. As the YSO begins to heat up its surroundings, and as energetic phenomena are initiated, material from the grain surfaces can go back into the gas phase, returning abundances to "normal." High-temperature chemical pathways and the addition of atomic species through grain disruption can lead to enhancements in specific mol-

\footnotetext{
${ }^{1}$ Astronomy Dept., University of Maryland, College Park, MD 20742.

${ }^{2}$ Division of Geological \& Planetary Sciences, California Institute of Technology 170-25, Pasadena, CA 91125.
}

ecules, relative to quiescent cloud material. Understanding which molecules are involved in these enhancements and when they occur is a current challenge in astrochemistry.

Ideally, we would like to construct a chemical-evolutionary sequence based on observed sources, which would help us to identify the underlying physical and chemical processes shaping the appearance of gas associated with the formation of a young stellar object. Thus far, single-dish surveys and interferometric studies of selected molecules have yielded indications of the comings and goings of molecules in the circumstellar environment, but not a complete or systematic picture (e.g., van Dishoeck et al. 1993). However, the identification of chemical "signposts" that mark different stages of development are beginning to be made and promise to be a useful tool for understanding the star formation process (e.g., McMullin et al. 1994; McMullin, Mundy, \& Blake 1993; Goldsmith et al. 1992; Blake et al. 1987; Plambeck \& Wright 1987).

The young stellar object IRAS 05338-0624 presents an excellent opportunity to explore the early circumstellar environment. It is a strong ( $\sim 490 \mathrm{Jy}$ at $100 \mu \mathrm{m})$, nearby $(450$ pc) far-infrared source with a steeply rising spectral energy distribution indicative of the earliest stages of pre-mainsequence evolution. $\mathrm{CO} J=1-0$ observations delineate a strong, bipolar outflow extended over a broad region (Wilking, Blackwell, \& Mundy 1990), further suggestive of early starforming processes. IRAS $05338-0624$ was detected at millimeter wavelengths by Wilking et al. (1989), revealing large amounts of cool dust, while recent studies in the near-infrared (Strom et al. 1989) failed to find a counterpart, suggesting the 
TABLE 1

OBSERVED TRANSITIONS: IRAS $05338-0624$

\begin{tabular}{|c|c|c|c|c|c|}
\hline Transition & Observatory & $\begin{array}{c}\text { Rest } \\
\text { Frequency } \\
(\mathrm{GHz})\end{array}$ & $\begin{array}{c}\text { Velocity } \\
\text { Resolution } \\
\left(\mathrm{km} \mathrm{s}^{-1}\right)\end{array}$ & $\begin{array}{l}\text { Beam } \\
\text { Size }\end{array}$ & $\begin{array}{c}\text { Typical } \\
\text { RMS Noise } \\
\text { (K) }\end{array}$ \\
\hline $\mathrm{H}^{13} \mathrm{CO}^{+} J=1 \rightarrow 0 \ldots \ldots \ldots$ & NRAO & 86.754 & 0.35 & $75^{\prime \prime}$ & 0.05 \\
\hline $\mathrm{C}^{34} \mathrm{~S} J=2 \rightarrow 1 \ldots \ldots \ldots \ldots$ & NRAO & 96.413 & 0.31 & 67 & 0.03 \\
\hline $\mathrm{CS} J=2 \rightarrow 1 \ldots \ldots \ldots \ldots$ & NRAO & 97.981 & 0.31 & 66 & 0.12 \\
\hline $\mathrm{C}^{18} \mathrm{O} J=1 \rightarrow 0 \ldots \ldots \ldots \ldots$ & NRAO & 109.782 & 0.27 & 59 & 0.11 \\
\hline $\mathrm{H}_{2} \mathrm{~S} 22_{20} \rightarrow 2_{11} \ldots \ldots \ldots \ldots$ & CSO & 216.710 & 0.07 & 34 & 0.06 \\
\hline $\mathrm{SiO} J=5 \rightarrow 4 \ldots \ldots \ldots \ldots$ & $\mathrm{CSO}$ & 217.105 & 0.07 & 34 & 0.06 \\
\hline $\mathrm{DCN} 3_{3} \rightarrow 2_{2} \ldots \ldots \ldots \ldots \ldots$ & CSO & 217.239 & 0.07 & 34 & 0.05 \\
\hline $\mathrm{C}^{18} \mathrm{O} J=2 \rightarrow 1 \ldots \ldots \ldots$ & CSO & 219.560 & 0.07 & 33 & 0.40 \\
\hline SO $5_{6} \rightarrow 4_{5} \ldots \ldots \ldots \ldots \ldots$ & CSO & 219.949 & 0.07 & 33 & 0.07 \\
\hline $\operatorname{CO} J=2 \rightarrow 1 \ldots \ldots \ldots \ldots$ & CSO & 230.538 & 0.06 & 32 & 0.24 \\
\hline $\mathrm{C}^{34} \mathrm{~S} J=5 \rightarrow 4 \ldots \ldots \ldots$ & CSO & 241.016 & 0.06 & 31 & 0.03 \\
\hline $\mathrm{CH}_{3} \mathrm{OH} 5_{14} \rightarrow 4_{14} \ldots \ldots \ldots$ & CSO & 241.767 & 0.60 & 31 & 0.14 \\
\hline $\mathrm{CH}_{3} \mathrm{OH} 5_{01} \rightarrow 4_{01} \ldots \ldots \ldots$ & $\mathrm{CSO}$ & 241.791 & 0.60 & 31 & 0.11 \\
\hline $\mathrm{CH}_{3} \mathrm{OH} 5_{13} \rightarrow 4_{13} \ldots \ldots \ldots$ & $\mathrm{CSO}$ & 241.879 & 0.60 & 31 & 0.09 \\
\hline $\mathrm{CH}_{3} \mathrm{OH} 5_{24} \rightarrow 4_{24} \cdots \ldots \ldots$ & CSO & 241.904 & 0.60 & 31 & 0.09 \\
\hline $\mathrm{CS} J=5 \rightarrow 4 \ldots \ldots \ldots \ldots$ & CSO & 244.936 & 0.06 & 30 & 0.16 \\
\hline $\mathrm{H}^{13} \mathrm{CN} J=3 \rightarrow 2 \ldots \ldots \ldots$ & CSO & 259.012 & 0.06 & 28 & 0.03 \\
\hline $\mathrm{C}_{2} \mathrm{H}_{44} \rightarrow 2_{33} \ldots \ldots \ldots \ldots$ & $\mathrm{CSO}$ & 262.004 & 0.06 & 28 & 0.07 \\
\hline $\mathrm{HCN} J=3 \rightarrow 2 \ldots \ldots \ldots$ & CSO & 265.886 & 0.05 & 28 & 0.09 \\
\hline $\mathrm{CH}_{3} \mathrm{OH} 7_{2} \rightarrow 6_{3} A^{-} \ldots \ldots$ & BIMA & 86.616 & 0.34 & $11 \times 10$ & $0.25^{\mathrm{a}}$ \\
\hline $\mathrm{H}^{13} \mathrm{CO}^{+} J=1 \rightarrow 0 \ldots \ldots$ & BIMA & 86.754 & 0.34 & $11 \times 10$ & $0.25^{\mathrm{a}}$ \\
\hline $\mathrm{SiO} J=2 \rightarrow 1 \ldots \ldots \ldots \ldots$ & BIMA & 86.847 & 0.34 & $11 \times 10$ & $0.25^{\mathrm{a}}$ \\
\hline $\mathrm{CH}_{3} \mathrm{OH} 8_{3} \rightarrow 9_{2} E \ldots \ldots \ldots$ & BIMA & 94.542 & 0.31 & $11 \times 9$ & $0.40^{\mathrm{a}}$ \\
\hline $\mathrm{SO}_{2} 7_{3,5} \rightarrow 8_{2,6} \ldots \ldots \ldots \ldots$ & BIMA & 97.702 & 0.30 & $11 \times 9$ & $0.22^{\mathrm{a}}$ \\
\hline $\mathrm{CS} J=2 \rightarrow 1 \ldots \ldots \ldots \ldots$ & BIMA & 97.981 & 0.30 & $11 \times 9$ & $0.22^{\mathrm{a}}$ \\
\hline
\end{tabular}

a Jy beam ${ }^{-1}$.

source is still very deeply embedded in its natal material. Other studies in dense gas tracers $\left(\mathrm{HCN}\right.$ and $\mathrm{HCO}^{+}$, Takaba et al. $1986 ; \mathrm{NH}_{3}$, Harju, Walmsley, \& Wouterloot 1991) support the presence of large amounts of cold, dense material, normally associated with YSOs.

We present here a detailed study of the $\lambda=1$ and $3 \mathrm{~mm}$ molecular emission from the IRAS $05338-0624$ region. The following results characterize the structure, excitation, and abundance patterns, over a range of scales, in the star-forming gas. In this work, we will use the term "condensation" to refer to the gas and dust distributed on $30^{\prime \prime}-60^{\prime \prime}$ scales $(0.07-0.13 \mathrm{pc})$ and "inner core" to refer to material concentrated on smaller scales and intimately associated with the young stellar object IRAS $05338-0624$.

\section{OBSERVATIONS}

\subsection{Molecular Line Observations}

Single-dish observations at $\lambda=2.7-3.1 \mathrm{~mm}$ were obtained with the $\mathrm{NRAO}^{3} 12 \mathrm{~m}$ telescope during 1992 May using the facility dual polarization receivers. Table 1 outlines the molecules, transitions, velocity resolutions, beam sizes, and typical rms noise levels. The system temperature of each polarization channel was $\sim 350 \mathrm{~K}$ single sideband. The spectrometer was configured to give two dual polarization 128 channel filterbanks with $100 \mathrm{kHz}$ resolution and a 128 channel filterbank with $30 \mathrm{kHz}$ resolution. Calibration was accomplished using the chopper wheel method, and the resulting data are reported as $T_{\mathrm{MB}}$, using a "corrected" main beam efficiency of $\eta_{m}^{*}=0.83$

\footnotetext{
${ }^{3}$ The National Radio Astronomy Observatory is operated by Associated Universities, Inc., under cooperative agreement with the National Science Foundation.
}

(Jewell 1988) at $98 \mathrm{GHz}$. Maps were acquired using absolute position switching to a reference position $47^{\prime} \mathrm{W}$ and $36^{\prime} \mathrm{S}$ of IRAS $05338-0624$.

Table 1 also outlines the observations obtained at $\lambda=1.1-$ $1.4 \mathrm{~mm}$ with the Caltech Submillimeter Observatory (CSO) ${ }^{4}$ using the facility $230 \mathrm{GHz}$ receiver in 1993 January. Weather conditions were characterized by $\tau(225 \mathrm{GHz}) \sim 0.15$; typical single sideband system temperatures ranged from 600 to 700 K. The facility 1024 channel acousto-optical spectrometers with 50 and $500 \mathrm{MHz}$ total bandwidth were utilized; the corresponding velocity resolutions, along with typical $T_{\text {rms }}$ for each observation, are given in Table 1 . The antenna temperatures, $T_{A}^{*}$, were converted to main beam temperatures using $\eta_{m}=$ 0.74 .

\subsection{Interferometer Observations}

Spectral line and continuum data toward IRAS 05338-0624 were obtained using the six-element BIMA $^{5}$ array between 1993 November and 1994 January. Two configurations of the 2048 channel digital correlator were utilized; the first to observe $\mathrm{SiO}, \mathrm{H}^{13} \mathrm{CO}^{+}$, and $\mathrm{SO}\left(0.34 \mathrm{~km} \mathrm{~s}^{-1}\right.$ resolution) and the second to observe $\mathrm{CS}, \mathrm{CH}_{3} \mathrm{OH}$, and $\mathrm{SO}_{2}$ $\left(0.3 \mathrm{~km} \mathrm{~s}^{-1}\right.$ resolution). In each case, the molecular lines were placed in the upper sideband to allow simultaneous continuum observations in the lower sideband at 86 and $95 \mathrm{GHz}$, respectively; frequencies, beam sizes, and typical noise values are given in Table 1 . The projected antenna spacings ranged from

\footnotetext{
${ }^{4}$ The Caltech Submillimeter Observatory is operated by the California Institute of Technology under funding from the U.S. National Science Foundation (no. AST-9313929).

${ }^{5}$ Berkeley, Illinois, and Maryland Association.
} 


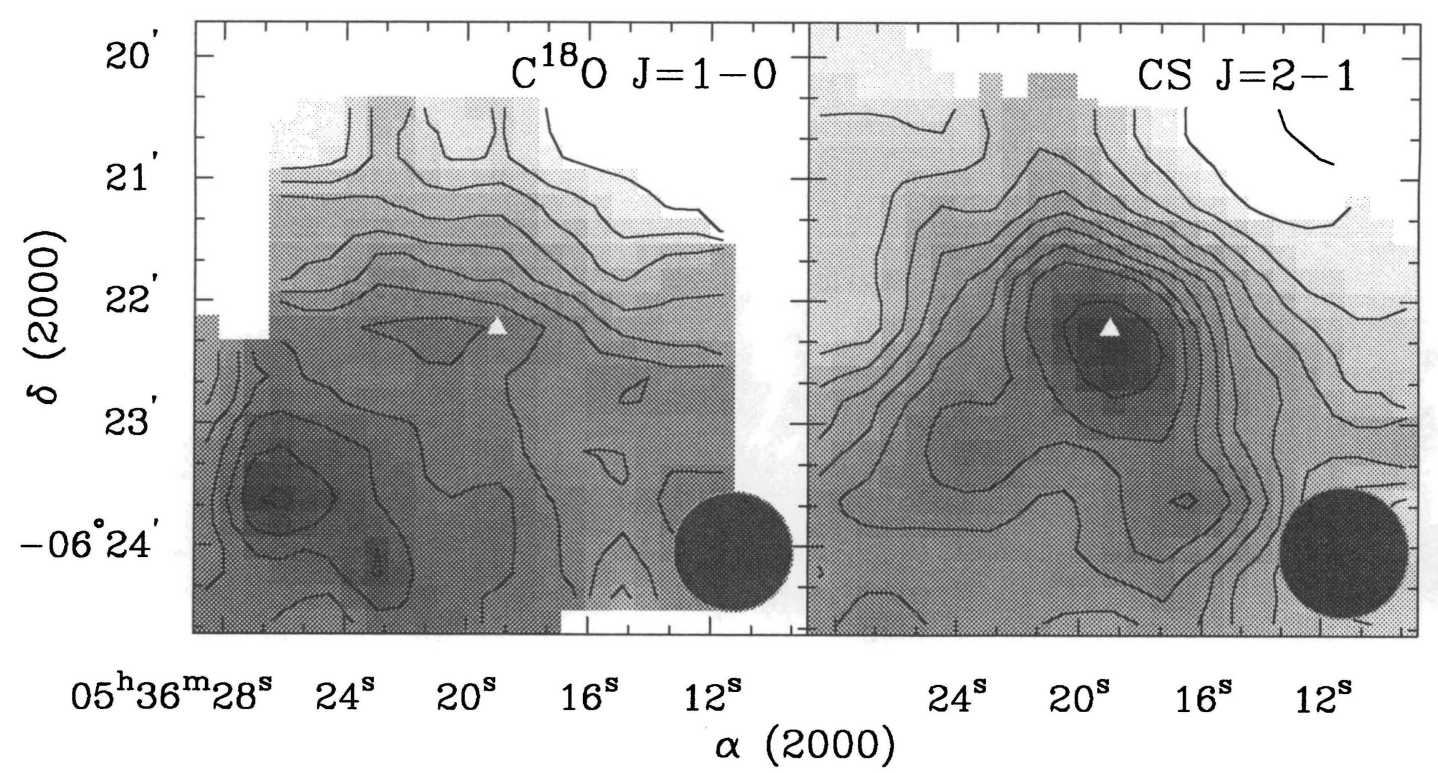

Fig. 1.-Integrated emission maps of $\mathrm{C}^{18} \mathrm{O} J=1-0$ and $\mathrm{CS} J=2-1$. The $\mathrm{C}^{18} \mathrm{O} J=1-0$ emission has been integrated from 4 to $10 \mathrm{~km} \mathrm{~s}{ }^{-1}$. The $\mathrm{CS} J=2-1$ emission has been integrated from 3 to $13 \mathrm{~km} \mathrm{~s}^{-1}$. For each panel, the contours are in steps of $0.5 \mathrm{~K} \mathrm{~km} \mathrm{~s}^{-1}$ (starting at $2 \mathrm{~K} \mathrm{~km} \mathrm{~s}^{-1}$ for $\mathrm{C}^{18} \mathrm{O}$ and $0.5 \mathrm{~K} \mathrm{~km} \mathrm{~s} \mathrm{~km}^{-1}$ for CS). The solid triangle marks the IRAS catalog position of IRAS $05338-0624: \alpha(\mathrm{J} 2000)=05^{\mathrm{h}} 36^{\mathrm{m}} 19^{\mathrm{s}} 0,0, \delta(\mathrm{J} 2000)=-06^{\circ} 22^{\prime} 13^{\prime \prime} .3$. Beams are shown in the lower right corner; beam sizes are given in Table 1 .

2 to $37 \mathrm{k} \lambda$. The resulting, naturally weighted synthesized beams are $11^{\prime \prime} \times 10^{\prime \prime}$ and $11^{\prime \prime} \times 9^{\prime \prime}$ at 86 and $98 \mathrm{GHz}$, respectively. The compact source $0530+135$ was used as a phase calibrator with a flux of $6.4 \mathrm{Jy}$ derived from planetary observations.

The data were reduced with the MIRIAD ${ }^{6}$ software package. A line-free continuum map was formed by averaging the channels in a correlator band showing no line emission. Spectral line maps were made by subtracting the dirty continuum maps from the dirty spectral line maps. The data were then CLEANed. No correction for primary beam taper has been applied.

\section{RESULTS}

\subsection{Overall Structure: $60^{\prime \prime}$ Scales}

Figure 1 shows the distribution of integrated emission in the $\mathrm{C}^{18} \mathrm{O} J=1-0$ (left panel) and CS $J=2-1$ (right panel) transitions. In the $\mathrm{C}^{18} \mathrm{O}$ map, IRAS 05338-0624 (the white triangle) sits within a plateau of emission $\left(\sim 7 \mathrm{~K} \mathrm{~km} \mathrm{~s}^{-1}\right)$ with no apparent peaking toward the source. The typical line widths around the source are $2.4 \mathrm{~km} \mathrm{~s}^{-1}$. Previous large beam observations of ${ }^{12} \mathrm{CO} J=1-0$ show an overall clumpy structure for this whole northern region of L1641 with no strict coincidence between emission peaks and IRAS point sources; however, $\mathrm{H}_{2}$ column density maps obtained from the ${ }^{12} \mathrm{CO}$ and ${ }^{13} \mathrm{CO}$ observations indicate a much stronger correspondence between $N_{\mathrm{H}_{2}}\left(\mathrm{H}_{2}\right.$ column density) peaks and IRAS sources (Takaba et al. 1986). There is little evidence of outflow activity seen in the $\mathrm{C}^{18} \mathrm{O}$ data, except toward the IRAS position where small excess emission redward of the line center is seen.

In contrast to the $\mathrm{C}^{\mathbf{1 8}} \mathrm{O}$ emission, the $\mathrm{CS} J=2-1$ emission strongly peaks on IRAS $05338-0624$, with an elongation at P.A. $=45^{\circ}$; observations of $\mathrm{HCO}^{+}$show a similar structure

\footnotetext{
${ }^{6}$ Multichannel Image Reconstruction, Image Analysis, and Display software developed by the Berkeley, Illinois Maryland Association.
}

(Fukui et al. 1988; Chen, Fukui, \& Yang 1992). Secondary peaks of CS emission are also seen to the southeast and southwest of the IRAS source, with one of the secondary peaks close to the peak in the $C^{18} \mathrm{O} J=1-0$ map (offset by $60^{\prime \prime}$ south). On larger scales, the CS $J=2-1$ emission is elongated southeast-northwest $\left(\right.$ P.A. $=135^{\circ}$ ) consistent with the large beam column density maps of Takaba et al. (1986). Maps of integrated $\mathrm{NH}_{3}(1,1)$ (Harju et al. 1991) are also similar in appearance to the CS $J=2-1$ map. Typical line widths for the CS $J=2-1$ transition are $2.4 \mathrm{~km} \mathrm{~s}^{-1}$, with an additional, weaker, broad component $\left(\sim 12 \mathrm{~km} \mathrm{~s}^{-1}\right)$ evident toward the IRAS position, indicative of outflow activity. A 9-point cross with $30^{\prime \prime}$ spacing was obtained in the $\mathrm{C}^{34} \mathrm{~S} J=2-1$ transition. The cross shows a slight peaking toward the continuum source position (falling off by roughly $20 \%$ at positions $30^{\prime \prime}$ off). The typical line width is $2 \mathrm{~km} \mathrm{~s}^{-1}$, slightly narrower than the CS $J=2-1$ lines. Figure 2 shows spectra taken toward the IRAS position for $\mathrm{C}^{18} \mathrm{O}, \mathrm{CS}$, and $\mathrm{C}^{34} \mathrm{~S}$.

\subsection{Overall Structure: $30^{\prime \prime}$ Scales}

The top left panel of Figure 3 shows a map of the integrated CS $J=5-4$ emission. The emission is centrally concentrated with a slight elongation of the highest contours in the east-west direction. Binning the redshifted and blueshifted emission yields the map in the bottom left panel, which shows the redshifted emission peaking $15^{\prime \prime}$ to the west of the continuum source; the weaker blueshifted emission appears to peak roughly at the continuum position with some more blueshifted emission toward the northwest. No self-absorptions are indicated in the line profiles. The morphology of the condensation in CS is similar to that seen in $\lambda=1.3 \mathrm{~mm}$ continuum emission (Walker, Adams, \& Lada 1990), though the CS emission is somewhat more extended.

The top center panel of Figure 3 shows the integrated SO $J_{\mathrm{K}}=6_{5}-5_{4}$ emission. Integrated SO peaks at a position $15^{\prime \prime} \mathrm{W}$ of the continuum source. The bottom center panel shows the 

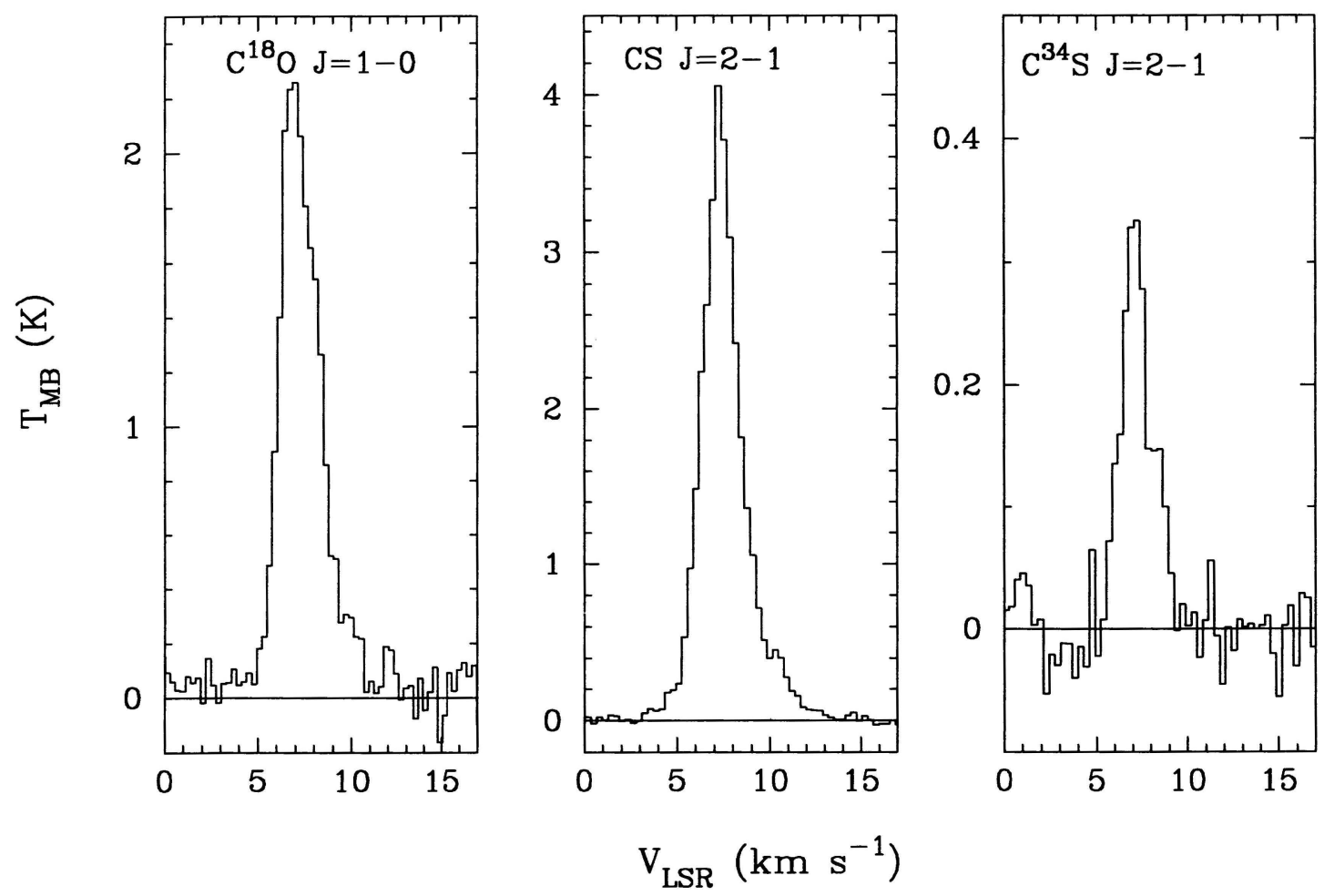

FIG. 2.-Spectra taken with NRAO $12 \mathrm{~m}$ telescope toward IRAS 05338-0624. The $T_{R}^{*}$ temperatures were converted to main beam brightness temperatures $\left(T_{\mathrm{MB}}\right)$ using $\eta=0.83$.

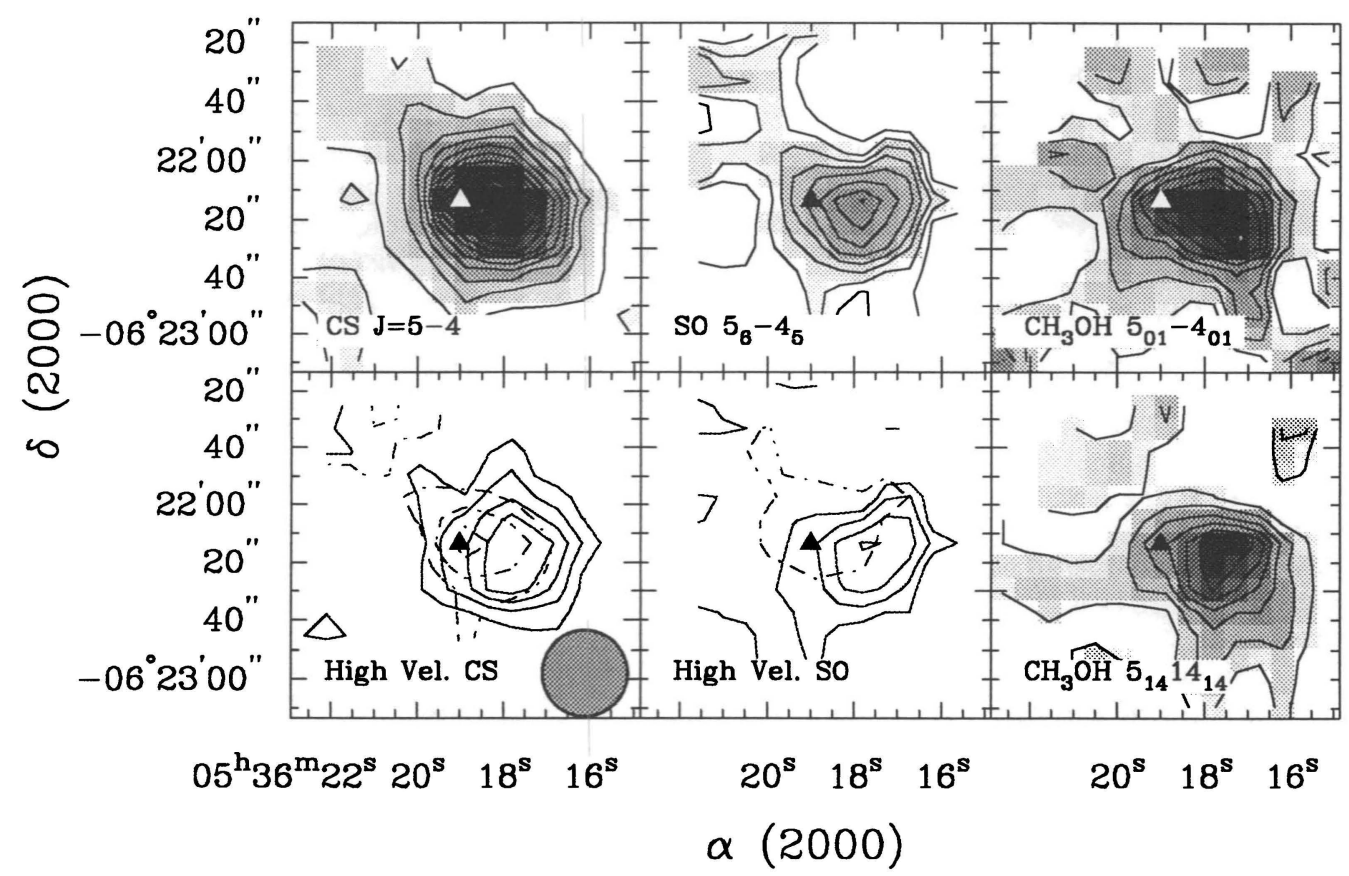

Fig. 3.-Integrated emission maps of CS $J=5-4$ (over 2-12 $\mathrm{km} \mathrm{s}^{-1}$ ), SO $5_{6}-4{ }_{5}\left(\right.$ over 2-20 km s${ }^{-1}$ ), and $\mathrm{CH}_{3} \mathrm{OH} 5_{01}-40_{01}\left(2-12 \mathrm{~km} \mathrm{~s}^{-1}\right)$ are shown in the top panels; $\mathrm{CH}_{3} \mathrm{OH} \mathrm{5}-44_{14}\left(2-12 \mathrm{~km} \mathrm{~s}^{-1}\right)$ is in the lower right panel. Contours are in steps of $0.5 \mathrm{~K} \mathrm{~km} \mathrm{~s}^{-1}$ for $\mathrm{CS}$ and $\mathrm{CH}_{3} \mathrm{OH}$; SO contours are in steps of $0.25 \mathrm{~K} \mathrm{~km}$ $\mathrm{s}^{-1}$ for the integrated emission. The bottom left panel displays the wing emission from CS $J=5-4$ where the blue-wing emission has been integrated from 3 to $6 \mathrm{~km}$ $\mathrm{s}^{-1}$ and the red-wing emission has been integrated from 9 to $12 \mathrm{~km} \mathrm{~s}^{-1}$; contours are in steps of $0.2 \mathrm{~K} \mathrm{~km} \mathrm{~s}^{-1}$. The bottom middle panel shows the blue (integrated from 3 to $6 \mathrm{~km} \mathrm{~s}^{-1}$ ) and the red-wing emission (integrated from 9 to $20 \mathrm{~km} \mathrm{~s}^{-1}$ ) from SO $5_{6}-4_{5}$; contours are in steps of 0.1 for the blue-wing emission and 0.25 for the red-wing emission. The triangles mark the location of IRAS 05338-0624. A representative beam is displayed in the lower left panel. 
redshifted and blueshifted SO emission which has a morphology similar to CS, with a redshifted peak at the $15^{\prime \prime} \mathrm{W}$ position and a weaker blueshifted peak closer to the continuum position. The top and bottom panels on the right of Figure 3 display the $\mathrm{CH}_{3} \mathrm{OH}$ emission as characterized by the $5_{14}-4_{14}$ and $5_{01}-4_{01}$ transitions. In both maps, the emission is compact and concentrated around the $15^{\prime \prime} \mathrm{W}$ position. Figure 4 shows a sequence of spectra taken at the continuum position and the $15^{\prime \prime} \mathrm{W}$ offset position. Spectra obtained at the western position show a clear enhancement in the redshifted emission in molecules such as $\mathrm{CH}_{3} \mathrm{OH}$, SO, and $\mathrm{HCN}$; $\mathrm{SiO}$ is seen only at the $15^{\prime \prime} \mathrm{W}$ position. DCN, $\mathrm{C}^{34} \mathrm{~S}$, and $\mathrm{C}^{18} \mathrm{O}$ show no obvious wing emission at the western position or the center position. The CS $J=5-4$ emission shows a clear red wing at the $15^{\prime \prime} \mathrm{W}$ position and a weaker red wing at the continuum peak. Table 2 lists the line parameters for the molecular species and transitions taken at the continuum position and the $15^{\prime \prime} \mathrm{W}$ position. The position of peak line temperature in each of the species shown in

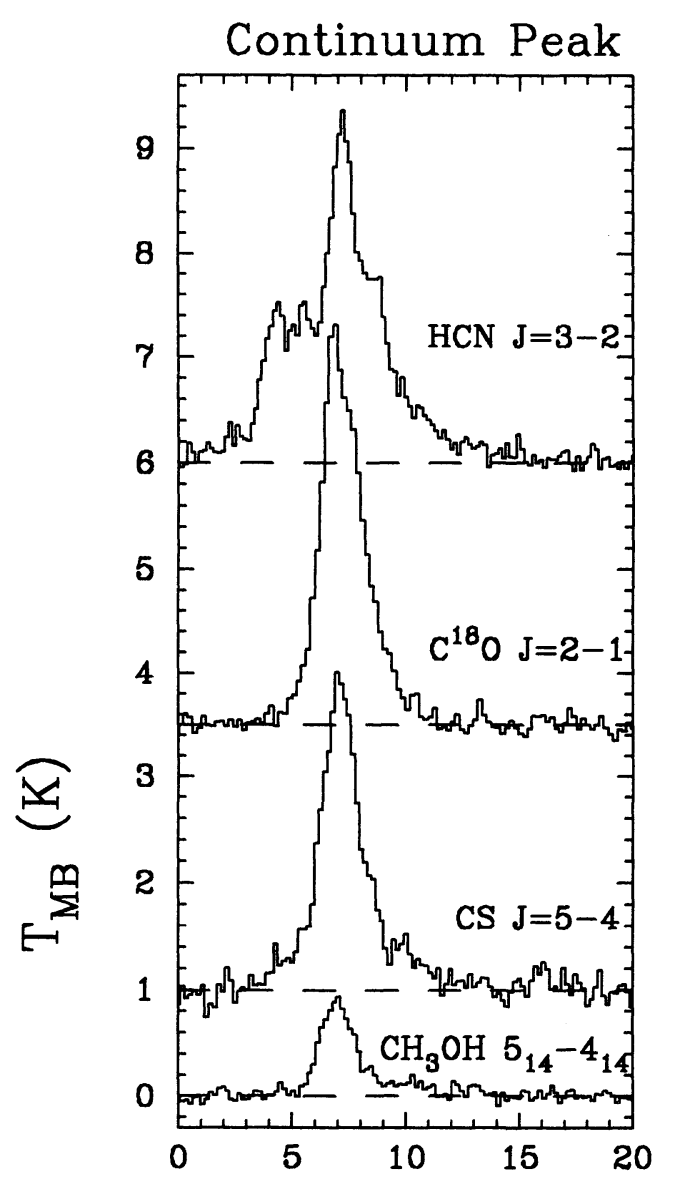

$15 " \mathrm{~W}$ of Continuum Peak
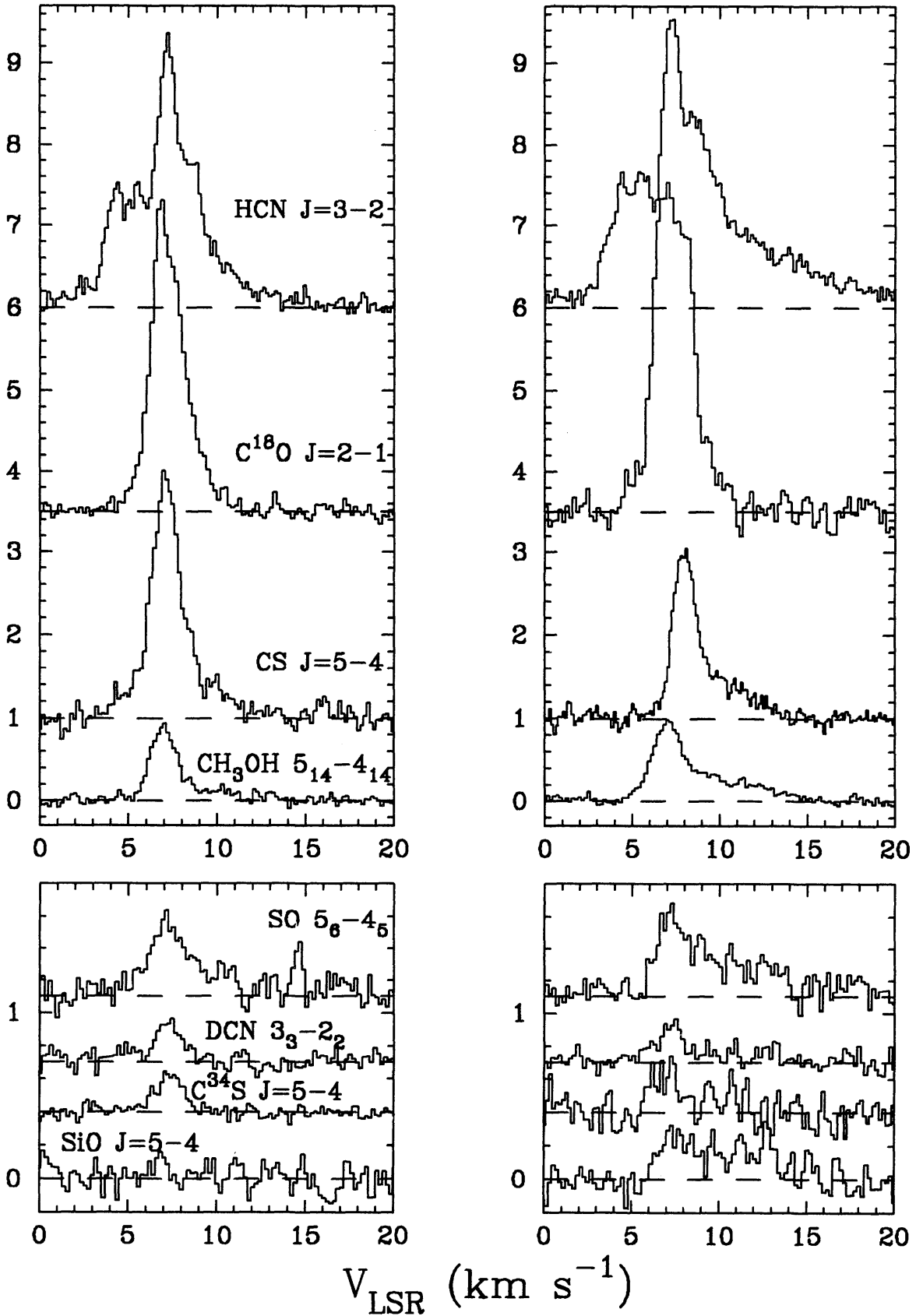

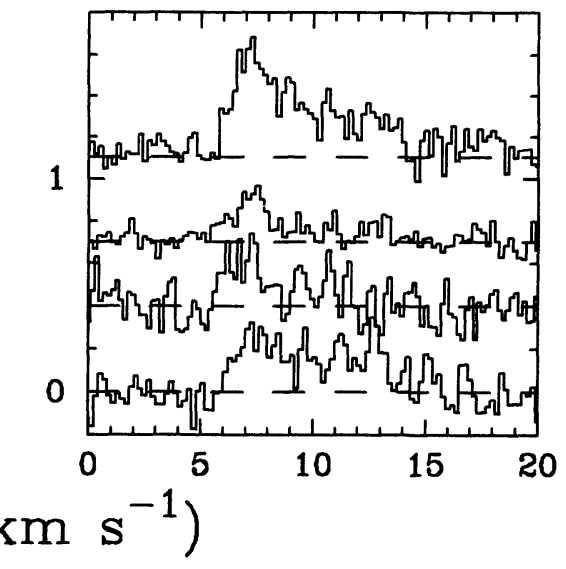

FIG. 4.-Spectra taken with the $230 \mathrm{GHz}$ receiver at the CSO toward IRAS 05338-0624 and the position of maximum red-wing emission, 15 " west. The antenna

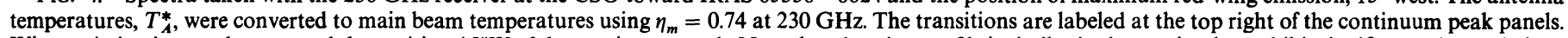

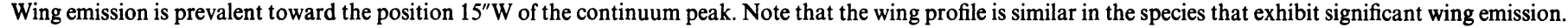


TABLE 2

Results of Spectral Line Survey

\begin{tabular}{|c|c|c|c|c|c|}
\hline Transition & Position & $\underset{(\mathrm{K})}{\operatorname{Peak} T_{\text {MB }}}$ & $\begin{array}{c}\text { RMS } \\
(\mathrm{K})\end{array}$ & $\begin{array}{c}V_{\mathrm{LSR}^{\mathrm{a}}} \\
\left(\mathrm{km} \mathrm{s}^{-1}\right)\end{array}$ & $\begin{array}{c}\Delta V^{\mathrm{b}} \\
\left(\mathrm{km} \mathrm{s} \mathrm{s}^{-1}\right)\end{array}$ \\
\hline $\mathrm{C}^{34} \mathrm{~S} J=2 \rightarrow 1 \ldots \ldots \ldots$ & $\left(0^{\prime \prime}, 0^{\prime \prime}\right)$ & 0.33 & 0.03 & 7.22 & 2.04 \\
\hline $\mathrm{C}^{34} \mathrm{~S} J=2 \rightarrow 1 \ldots \ldots \ldots$ & $(-15,0)$ & 0.29 & 0.04 & 7.68 & 1.75 \\
\hline $\operatorname{CS} J=2 \rightarrow 1 \ldots \ldots \ldots \ldots$ & $(0,0)$ & 4.06 & 0.03 & 7.43 & 2.41 \\
\hline $\mathrm{CS} J=2 \rightarrow 1 \ldots \ldots \ldots \ldots$ & $(-15,0)$ & 3.07 & 0.12 & 7.42 & 2.33 \\
\hline $\mathrm{C}^{18} \mathrm{O} J=1 \rightarrow 0 \ldots \ldots \ldots$ & $(0,0)$ & 2.26 & 0.08 & 7.25 & 2.43 \\
\hline $\mathrm{C}^{18} \mathrm{O} J=1 \rightarrow 0 \ldots \ldots \ldots$ & $(-15,0)$ & 2.21 & 0.14 & 7.04 & 2.19 \\
\hline $\mathrm{SiO} J=5 \rightarrow 4 \ldots \ldots \ldots \ldots$ & $(0,0)$ & $<0.24$ & 0.12 & $\ldots$ & $\ldots$ \\
\hline $\mathrm{SiO} J=5 \rightarrow 4 \ldots \ldots \ldots \ldots$ & $(-15,0)$ & 0.28 & 0.06 & 11.74 & 7.40 \\
\hline DCN $3_{3} \rightarrow 2_{2} \ldots \ldots \ldots \ldots$ & $(0,0)$ & 0.31 & 0.08 & 6.99 & 1.90 \\
\hline $\mathrm{DCN} 3_{3} \rightarrow 2_{2} \ldots \ldots \ldots \ldots$ & $(-15,0)$ & 0.29 & 0.08 & 7.57 & 2.38 \\
\hline $\mathrm{CO} J=2 \rightarrow 1 \ldots \ldots \ldots \ldots$ & $(0,0)$ & 22.4 & 0.24 & 8.22 & 5.92 \\
\hline $\mathrm{CO} J=2 \rightarrow 1 \ldots \ldots \ldots \ldots$ & $(-15,0)$ & 23.5 & 0.24 & 8.48 & 5.76 \\
\hline $\mathrm{C}^{18} \mathrm{O} J=2 \rightarrow 1 \ldots \ldots \ldots$ & $(0,0)$ & 3.87 & 0.56 & 7.19 & 2.25 \\
\hline $\mathrm{C}^{18} \mathrm{O} J=2 \rightarrow 1 \ldots \ldots \ldots$ & $(-15,0)$ & 4.17 & 0.71 & 7.36 & 2.54 \\
\hline SO $5_{6} \rightarrow 4_{5} \ldots \ldots \ldots \ldots \ldots$ & $(0,0)$ & 0.65 & 0.10 & 7.48 & 2.67 \\
\hline SO $5_{6} \rightarrow 4_{5} \ldots \ldots \ldots \ldots \ldots$ & $(-15,0)$ & 0.65 & 0.10 & 8.90 & 6.24 \\
\hline $\mathrm{C}^{34} \mathrm{~S} J=5 \rightarrow 4 \ldots \ldots \ldots$ & $(0,0)$ & 0.29 & 0.04 & 7.22 & 1.84 \\
\hline$C^{34} S J=5 \rightarrow 4 \ldots \ldots \ldots$ & $(-15,0)$ & 0.41 & 0.12 & 7.95 & 1.90 \\
\hline $\mathrm{CH}_{3} \mathrm{OH} 5_{14} \rightarrow 4_{14} \ldots \ldots$. & $(0,0)$ & 0.96 & 0.18 & 7.22 & 1.89 \\
\hline $\mathrm{CH}_{3} \mathrm{OH} 5_{14} \rightarrow 4_{14} \ldots \ldots$ & $(-15,0)$ & 1.01 & 0.22 & 7.73 & 3.06 \\
\hline $\mathrm{CH}_{3} \mathrm{OH} 5_{01} \rightarrow 4_{01} \ldots \ldots$ & $(0,0)$ & 0.95 & 0.14 & 7.77 & 6.09 \\
\hline $\mathrm{CH}_{3} \mathrm{OH} 5_{01} \rightarrow 4_{01} \ldots \ldots$ & $(-15,0)$ & 1.44 & 0.15 & 7.18 & 4.63 \\
\hline $\mathrm{CH}_{3} \mathrm{OH} 5_{13} \rightarrow 4_{13} \cdots \cdots$ & $(0,0)$ & 0.27 & 0.12 & $\ldots$ & $\ldots$ \\
\hline $\mathrm{CH}_{3} \mathrm{OH} 5_{24} \rightarrow 4_{24} \ldots \ldots$ & $(0,0)$ & 0.30 & 0.12 & $\ldots$ & $\ldots$ \\
\hline $\mathrm{CH}_{3} \mathrm{OH} 5_{24} \rightarrow 4_{24} \ldots \ldots$ & $(-15,0)$ & 0.51 & 0.13 & 6.75 & 2.52 \\
\hline $\mathrm{CS} J=5 \rightarrow 4 \ldots \ldots \ldots \ldots$ & $(0,0)$ & 3.10 & 0.15 & 7.19 & 2.18 \\
\hline $\mathrm{CS} J=5 \rightarrow 4 \ldots \ldots \ldots \ldots$ & $(-15,0)$ & 2.11 & 0.10 & 7.70 & 2.70 \\
\hline $\mathrm{H}^{13} \mathrm{CN} J=3 \rightarrow 2 \ldots \ldots$ & $(0,0)$ & 0.31 & 0.05 & 7.23 & 2.07 \\
\hline $\mathrm{C}_{2} \mathrm{H}_{44} \rightarrow 2_{33} \ldots \ldots \ldots \ldots$ & $(0,0)$ & 2.71 & 0.10 & 6.10 & 4.23 \\
\hline $\mathrm{HCN} J=3 \rightarrow 2 \ldots \ldots \ldots$ & $(0,0)$ & 3.39 & 0.13 & 7.11 & 4.79 \\
\hline $\mathrm{HCN} J=3 \rightarrow 2 \ldots \ldots \ldots$ & $(-15,0)$ & 3.61 & 0.12 & 8.01 & 6.47 \\
\hline
\end{tabular}

a Derived from centroid.

b Determined from one-component Gaussian fit to line core.

Figure 3 is coincident with the IRAS position; the elongation in the integrated maps arises from broad line wings seen toward the $15^{\prime \prime} \mathrm{W}$ position. In general, spectra taken at the western position display broader lines and have their centroids shifted to slightly higher velocities.

\subsection{Compact Structure: $10^{\prime \prime}$ Scales}

The millimeter wavelength interferometric observations provide a more detailed portrait of the gas distribution. The upper left panel in Figure 5 displays the CS $J=2 \rightarrow 1$ integrated intensity map composed of all channels with $4 \sigma$ or greater emission. The emission is elongated at a position angle of $45^{\circ}$, with peaks at the continuum position and at the position $14^{\prime \prime}$ west and $8^{\prime \prime}$ south of the continuum position. The negative contours on the map are artifacts of large scale emission which was poorly sampled by the interferometer. The lower left panel displays a superposition of the $\lambda=3 \mathrm{~mm}$ continuum emission (gray scale and dark contours) with the redshifted (gray contours) and blueshifted (dashed contours) CS emission. There is a clear indication of bi-polar outflow centered on the continuum position, oriented at a position angle of $45^{\circ}$, as previously suggested by $\mathrm{CO}$ observations of Fukui et al. (1988).

$\mathrm{H}^{13} \mathrm{CO}^{+} J=1-0$ emission is shown in the upper right panel of Figure 5. The emission is also elongated and oriented at roughly a $45^{\circ}$ angle. In this case, however, there is no indication of outflow activity; the line widths are only $\sim 2 \mathrm{~km} \mathrm{~s}^{-1}$. The map shows two discrete peaks in the $\mathrm{H}^{13} \mathrm{CO}^{+}$emission distribution: one is located slightly offset from the $\lambda=3 \mathrm{~mm}$ continuum position, while the other is to the east of the location of the peak CS blue-wing emission in the lower left panel of Figure 5; this could represent a clump of quiescent material which is interacting with the blue outflow lobe akin to the $\mathrm{HCO}^{+}$emission seen by Rudolph \& Welch (1988). In contrast, the $\mathrm{SiO} J=2-1$ emission, which is shown in the lower right panel of Figure 5, peaks at the location of the peak CS redwing emission. The line is broad $\left(\sim 10 \mathrm{~km} \mathrm{~s}^{-1}\right)$ and peaks around $V_{\mathrm{LSR}}=12 \mathrm{~km} \mathrm{~s}^{-1}$, significantly offset from the $V_{\mathrm{LSR}}$ of the core, $7 \mathrm{~km} \mathrm{~s}^{-1}$. The $\mathrm{SiO}$ emission extends to the northeast, toward the continuum position. At the continuum peak the emission is even further offset from the condensation velocity, peaking around $16 \mathrm{~km} \mathrm{~s}^{-1}$.

The $\mathrm{CH}_{3} \mathrm{OH}$ and $\mathrm{SO}_{2}$ transitions observed with the BIMA array were not detected at the $3 \sigma$ level. Both methanol transitions observed with the interferometer are relatively high above the ground (131 and $102 \mathrm{~K}$ for the $8_{3,3} \rightarrow 9_{23}$ and $7_{2,2} \rightarrow 6_{3,2}$ transitions, respectively) and have weaker line strengths by a factor of at least 10 than the transitions observed at the CSO. Additionally, other transitions of methanol with similar energy requirements and larger line strengths than these transitions were within the $500 \mathrm{MHz}$ band at the CSO and were only weakly detected $(2-3 \sigma)$. Hence, it is not surprising that neither $\mathrm{CH}_{3} \mathrm{OH}$ transition was detected. The $\mathrm{SO}_{2}$ maps show no obvious peak; however, spectra taken toward the location of the peak CS wing emission are suggestive of faint emission ( $2 \sigma$ level). The spectrum taken toward the 


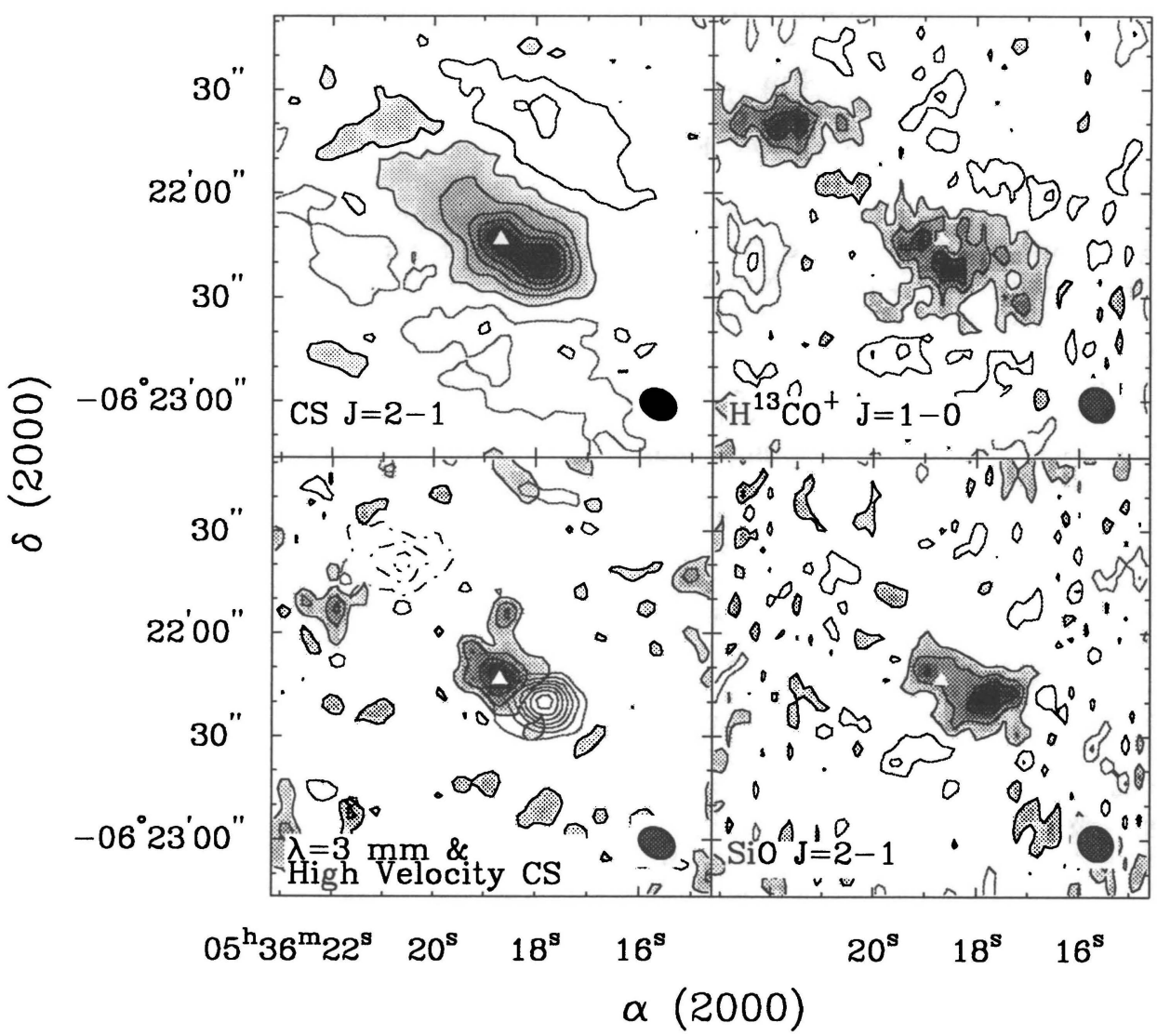

Fig. 5.-Multipanel map of the integrated emission from the BIMA array observations. The solid white triangle marks the location of the peak continuum emission: $\alpha(\mathrm{J} 2000)=05^{\mathrm{h}} 36^{\mathrm{m}} 18^{\mathrm{s}} 7, \delta(\mathrm{J} 2000)=-06^{\circ} 22^{\prime} 9^{\prime \prime} .5$. For the CS $\mathrm{J}=2 \rightarrow 1$ map in the upper left panel, the contours are in steps of $\pm 6 \mathrm{Jy}^{2}$ beam ${ }^{-1} \mathrm{~km} \mathrm{~s}^{-1}$, starting with $3 \mathrm{Jy}$ beam ${ }^{-1} \mathrm{~km} \mathrm{~s}^{-1}$, with a peak of $38 \mathrm{Jy}_{\text {beam }}{ }^{-1} \mathrm{~km} \mathrm{~s}^{-1}$. In the lower left panel, the continuum emission contours (dark solid lines) are in steps of \pm 20 $\mathrm{mJy}$ beam ${ }^{-1}$ with a peak of $0.103 \mathrm{Jy}_{\text {beam }}{ }^{-1}$. The gray contours are the redshifted CS $J=2 \rightarrow 1$ emission binned from 10.3 to $15.7 \mathrm{~km} \mathrm{~s}^{-1}$; the dash-dot gray lines are the blueshifted CS $J=2 \rightarrow 1$ emission binned from 1.3 to $4.3 \mathrm{~km} \mathrm{~s}^{-1}$. In the lower right panel, the $\mathrm{H}^{13} \mathrm{CO}^{+} J=1-0$ contours are in steps of $\pm 1 \mathrm{Jy}$ beam ${ }^{-1} \mathrm{~km}$ $\mathrm{s}^{-1}$ with a peak of $4.4 \mathrm{Jy}$ beam ${ }^{-1} \mathrm{~km} \mathrm{~s}^{-1}$. The $\mathrm{SiO} J=2-1$ has contours in steps of $2 \mathrm{Jy}$ beam $\mathrm{km} \mathrm{s}^{-1}$ with a peak of $8.3 \mathrm{Jy}$ beam $\mathrm{km} \mathrm{s}^{-1} \mathrm{~km}^{-1}$.

continuum peak shows a marginal absorption at the cloud $V_{\text {LSR }}$, also at the $2 \sigma$ level.

\section{PHYSICAL PROPERTIES OF IRAS 05338-0624}

In this section we will derive the density mass, column density, and abundances for the gas associated with IRAS 05338-0624. For these calculations, the condensation and inner core are identified as distinct structural and kinematic components; the condensation is represented by $30^{\prime \prime}-60^{\prime \prime}$ resolution observations while the inner core properties are probed with the $10^{\prime \prime}$ resolution interferometer observations. For both, the FWZI of the $\mathrm{C}^{18} \mathrm{O}$ emission $\left(5-10 \mathrm{~km} \mathrm{~s}^{-1}\right)$ is the velocity range of integration. Any emission beyond this velocity range is interpreted as resulting from interaction of the YSO with surrounding material.

\subsection{Gas Density in the Condensation}

A large velocity gradient (LVG) radiative transfer code was used to model the single-dish data in order to provide an estimate of the characteristic density. The measured line temperatures for the $C^{34} S J=2-1$ and $C^{34} S J=5-4$ transitions are 0.33 and $0.29 \mathrm{~K}$ (smoothing the $J=5-4$ data to match the $J=2-1$ resolution yields a $T_{\mathrm{MB}}=0.062 \mathrm{~K}$ ). Using an adopted temperature of $42 \mathrm{~K}$ (Walker et al. 1990), we derive a best-fit density of $2 \times 10^{5} \mathrm{~cm}^{-3}$ and a column density for $\mathrm{C}^{34} \mathrm{~S}$ of $2.5 \times 10^{12} \mathrm{~cm}^{-2}$. Assuming a terrestrial ratio of ${ }^{32} \mathrm{~S} /$ ${ }^{34} \mathrm{~S}=22.5$, we obtain a CS column density of $5.6 \times 10^{13} \mathrm{~cm}^{-2}$. Temperature variations of $10 \mathrm{~K}$ result in density and column density uncertainties of $\sim 40 \%$ and $\sim 5 \%$, respectively. Based on $\chi^{2}$ fitting of the data, we estimate $2 \sigma$ uncertainties of $40 \%-$ $50 \%$. These uncertainty estimates do not take into account the possibility of foreground, low-density material or density gradients along the line of sight that could significantly alter the parameters. We adopt an overall uncertainty of a factor of 2 for our density and CS column density estimates; this uncertainty could be even larger if there are large amounts of foreground material.

\subsection{Mass}

Estimates for the mass can be derived independent of the molecular line data based on the integrated millimeter continuum flux (e.g., Hildebrand 1983; Beckwith \& Sargent 1991). The value of the mass opacity $\left(\kappa_{v}\right)$ at millimeter wavelengths is poorly constrained and represents the major uncertainty in this mass calculation. We adopt the standard power-law representation, $\kappa_{v}=\kappa_{0}\left(v / v_{0}\right)^{\beta}$, normalized to the mass opacity coefficient $\kappa_{0}=0.1 \mathrm{~cm}^{2} \mathrm{~g}^{-1}$ at $v_{0}=1.2 \times 10^{12} \mathrm{~Hz}(\lambda=250 \mu \mathrm{m})$ from Hildebrand (1983). Values of both $\kappa_{0}$ and $\beta$ are uncertain and can depend on grain material and grain size distribution 
(Miyake \& Nakagawa 1993; Beckwith \& Sargent 1991). The value of $\kappa_{0}$ is estimated to be known only to within a factor of 2-3 based on comparison of dust opacities used in previous papers (Draine 1990) and based on calculations of opacities for various grain distributions (Miyake \& Nakagawa 1993). From calculations of grain properties, the value of $\beta$ could range from 0 , for grains that are large compared to the wavelength, to 2 for spherical grains that are small compared to the wavelength. For wavelengths longward of $\lambda=250 \mu \mathrm{m}$, it is expected that $\beta=2$ is appropriate for interstellar dust (e.g., Draine \& Lee 1984), and there is observational evidence to support $\beta \approx 2$ on large size scales (Chini, Krugel, \& Kreysa 1986; Hildebrand 1983; Schwartz 1982). On the small scales associated with individual young and forming stars, observational determinations of $\beta$ range from -1 to 2 , with values around $\beta=1$ most characteristic (Weintraub, Sandell, \& Duncan 1991; Beckwith \& Sargent 1991; Walker et al. 1990; Woody et al. 1989; Whittet 1988). The change in $\beta$ from 2 in the broad interstellar medium to $\sim 1$ in circumstellar nebulae is commonly interpreted as due to particle growth and compositional changes in the circumstellar environment (e.g., Beckwith \& Sargent 1991).

For our mass estimates, and later our $\mathrm{H}_{2}$ column density estimates, the important quantity is the mass opacity at millimeter wavelengths. We choose to fix $\kappa_{0}$ and parameterize the mass opacity at millimeter wavelengths through the value of the power-law index, $\beta$. Based on the above discussion, we adopt $\beta=2$ for the condensation scale and $\beta=1$ for the inner core in our calculations. The mass opacity coefficient corresponding to $\beta=2$ is a factor of 5 smaller at $\lambda=1.3 \mathrm{~mm}$, and a factor of 12 smaller at $\lambda=3 \mathrm{~mm}$, than for $\beta=1$; the resulting mass and $\mathrm{H}_{2}$ column density estimates are the corresponding factors larger for $\beta=2$ compared to $\beta=1$.

Using the integrated flux from Walker et al. (1990, $2.7 \mathrm{Jy}$ at $\lambda=1.3 \mathrm{~mm}$ ), we derive a mass for the condensation of $10.2 M_{\odot}$ $(\beta=2)$. The mass of the condensation may be estimated in several other ways. Choosing a characteristic diameter of $\sim 60^{\prime \prime}$ $(0.13 \mathrm{pc}$ at a distance of $450 \mathrm{pc})$ based on the CS emission distribution, we derive a mass of $15.9 M_{\odot}$ assuming a spherical geometry and a uniform density as derived from the fitted $\mathrm{C}^{34} \mathrm{~S}$ transitions. With this same diameter we derive a virial mass for a uniform density sphere of $40 M_{\odot}$, using a characteristic line width of $2 \mathrm{~km} \mathrm{~s}^{-1}$ as determined from $\mathrm{C}^{34} \mathrm{~S}$ observations. Previous estimates range from $27 M_{\odot}$ by Chen et al. (1992) to $150 M_{\odot}$ by Takaba et al. (1986); the lower value is based on the mass of just the HCN core while the higher value is based on an estimate of the total mass of larger scale gas distribution. We adopt the virial mass of $40 M_{\odot}$ as a characteristic mass for the dense condensation around IRAS 05338-0624.

A mass estimate may be obtained for the inner core from the $\lambda=3 \mathrm{~mm}$ continuum observations. The total observed flux was $176 \mathrm{mJy}$ at $\lambda=3 \mathrm{~mm}$ yielding a mass of $1.9 M_{\odot}(\beta=1)$. The derived mass is consistent with previous estimates based on high-resolution observations (1.3 $M_{\odot}$, Walker et al. 1990; $4.3 M_{\odot}$, Wilking et al. 1989).

The difference in the mass derived for the inner core and that for the overall condensation is directly affected by the choice of the power-law index for the dust emissivity. If a uniform dust emissivity law is applied to both the inner core and the condensation, the mass derived for the inner core is approximately the same as the mass derived for the condensation using the $\lambda=1.3 \mathrm{~mm}$ emission; this result is not supported by the extended emission distributions of both dense gas tracers and $\lambda=1.3 \mathrm{~mm}$ dust emission. Adopting a mass of $2 M_{\odot}$ for the inner core, roughly $5 \%$ of the condensation is found intimately associated with the YSO.

\subsection{Column Density}

The $\mathrm{H}_{2}$ column density and the column density for the various molecular species may be obtained directly from the observed fluxes (e.g., Hildebrand 1983; Blake et al. 1987). Table 3 lists the integrated emission for the different species, and Table 4 lists the molecular column densities derived. As noted in Table 4, optically thin species were used to estimate the column densities of common species whenever possible.

\subsection{Molecular Fractional Abundances}

Fractional abundances for the observed molecular species are derived from the ratio of the column density of the species, $N_{x}$, to $N_{\mathrm{H}_{2}}$. The resulting values are provided in Table 5 . Values cited for the condensation are based on the $N_{\mathbf{H}_{2}}$ column density derived from the $\lambda=1.25 \mathrm{~mm}$ continuum emission using a $v^{2}$ emissivity; using a $v^{1}$ emissivity would increase the

TABLE 3

INTEGRATED MOLECULAR EMISSION TOWARD IRAS 05338-0624

\begin{tabular}{|c|c|c|c|c|}
\hline \multirow[b]{2}{*}{ Transition } & \multicolumn{2}{|c|}{$\begin{array}{c}\text { AMBIENT GAS } \\
\text { AT } I R A S \text { POSITION }\end{array}$} & \multicolumn{2}{|c|}{$\begin{array}{c}\text { SHOCKed Gas } \\
\text { AT PEAK Wing Position }\end{array}$} \\
\hline & $\begin{array}{l}\text { Condensation } \\
\left(\mathrm{K} \mathrm{km} \mathrm{s}^{-1}\right)\end{array}$ & $\begin{array}{c}\text { Inner Core } \\
\left(\mathrm{Jy} \mathrm{beam}^{-1} \mathrm{~km} \mathrm{~s}^{-1}\right)\end{array}$ & $\begin{array}{l}\text { 30" Resolution } \\
\left(\mathrm{K} \mathrm{km} \mathrm{s}^{-1}\right)\end{array}$ & $\begin{array}{c}10^{\prime \prime} \text { Resolution } \\
\left(\mathrm{Jy} \mathrm{beam}^{-1} \mathrm{~km} \mathrm{~s}^{-1}\right)\end{array}$ \\
\hline $\operatorname{CO} J=2-1 \ldots \ldots \ldots \ldots$ & 127. & $\ldots$ & 46.2 & $\ldots$ \\
\hline $\mathrm{CS} J=5-4 \ldots \ldots \ldots \ldots \ldots$ & 6.58 & $\cdots$ & 1.36 & $\cdots$ \\
\hline$C^{34} S J=5-4 \ldots \ldots \ldots$ & 0.5 & $\ldots$ & $\ldots$ & $\ldots$ \\
\hline $\operatorname{CS} J=2-1 \ldots \ldots \ldots \ldots$ & 9.3 & 25.1 & 0.5 & 12.1 \\
\hline $\mathrm{C}^{34} \mathrm{~S} J=2-1 \ldots \ldots \ldots \ldots$ & 0.7 & $\ldots$ & $\ldots$ & $\ldots$ \\
\hline $\mathrm{SiO} J=5-4 \ldots \ldots \ldots \ldots$ & $<0.2$ & $\ldots$ & 0.75 & $\ldots$ \\
\hline $\mathrm{SiO} J=2-1 \ldots \ldots \ldots \ldots$ & $\ldots$ & $<3.3$ & $\ldots$ & 6.7 \\
\hline $\mathrm{CH}_{3} \mathrm{OH} 5_{14}-4_{14} \ldots \ldots \ldots$ & 1.85 & $\ldots$ & 0.79 & $\ldots$ \\
\hline HCN $J=3-2 \ldots \ldots \ldots \ldots$ & 8.73 & $\cdots$ & $3.1^{\mathrm{c}}$ & $\ldots$ \\
\hline $\mathrm{H}^{13} \mathrm{CN} J=3-2 \ldots \ldots \ldots$ & 0.6 & $\ldots$ & $\ldots$ & $\ldots$ \\
\hline $\mathrm{DCN} J=3-2 \ldots \ldots \ldots$ & 0.31 & $\ldots$ & $\ldots$ & $\ldots$ \\
\hline SO $5_{6}-4_{5} \ldots \ldots \ldots \ldots \ldots$ & 1.28 & $\ldots$ & 0.74 & $\ldots$ \\
\hline $\mathrm{H}^{13} \mathrm{CO}^{+} J=1-0 \ldots \ldots$ & 1.48 & 4.4 & $\ldots$ & $<1$ \\
\hline
\end{tabular}

a Velocity interval from 5 to $10 \mathrm{~km} \mathrm{~s}^{-1}$.

b Velocity interval from 10 to $15 \mathrm{~km} \mathrm{~s}^{-1}$.

c Emission from the hyperfine component $F=1-1$ has been subtracted. 
TABLE 4

ProperTIES OF IRAS 05338-0624

\begin{tabular}{|c|c|}
\hline Quantity & Value \\
\hline \multicolumn{2}{|l|}{ Condensation } \\
\hline 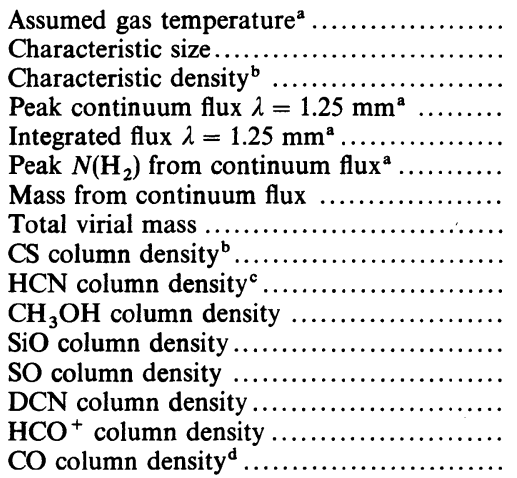 & $\begin{array}{c}42 \mathrm{~K} \\
60^{\prime \prime}(0.13 \mathrm{pc}) \\
2 \times 10^{5} \mathrm{~cm}^{-3} \\
2.05 \mathrm{Jy} \mathrm{beam}-1 \\
2.67 \mathrm{Jy} \\
8.3(1.7) \times 10^{22} \mathrm{~cm}^{-2} \\
10 M_{\odot} \\
40 M_{\odot} \\
5.6 \times 10^{13} \mathrm{~cm}^{-2} \\
3.2 \times 10^{13} \mathrm{~cm}^{-2} \\
2.2 \times 10^{14} \mathrm{~cm}^{-2} \\
<2.1 \times 10^{11} \mathrm{~cm}^{-2} \\
1.3 \times 10^{13} \mathrm{~cm}^{-2} \\
7.0 \times 10^{11} \mathrm{~cm}^{-2} \\
3.5 \times 10^{14} \mathrm{~cm}^{-2} \\
1.2 \times 10^{18} \mathrm{~cm}^{-2}\end{array}$ \\
\hline
\end{tabular}

Inner Core

Peak continuum position (J2000)

Integrated continuum flux $\lambda=3.1 \mathrm{~mm} \ldots \ldots$.

Peak $N\left(\mathrm{H}_{2}\right)$ from continuum flux

Mass from continuum flux ................

SiO column density.

$\mathrm{HCO}^{+}$column density ${ }^{\mathrm{e}}$

CS column density ........................

$05^{\mathrm{h}} 36^{\mathrm{m}} 18^{\mathrm{s}} .68-06^{\circ} 22^{\prime} 9^{\prime \prime} 5$

$103 \mathrm{mJy}^{-1}$ beam $^{-1}$

$176 \mathrm{mJy}$

$$
\begin{aligned}
& 8.5 \times 10^{22} \mathrm{~cm}^{-2} \\
1.9 M_{\odot} & \\
< & 2.4 \times 10^{13} \mathrm{~cm}^{-2} \\
& 1.6 \times 10^{15} \mathrm{~cm}^{-2} \\
& 2.8 \times 10^{14} \mathrm{~cm}^{-2}
\end{aligned}
$$

a Based on Walker et al. 1990.

${ }^{b}$ Derived from LVG model; inferred from $\mathrm{C}^{34} \mathrm{~S}$.

${ }^{\circ}$ Inferred from $\mathrm{H}^{13} \mathrm{CN}$; assumes ${ }^{12} \mathrm{C} /{ }^{13} \mathrm{C}=60$.

${ }^{\mathrm{d}}$ Inferred from $\mathrm{C}^{18} \mathrm{O}$; assumes ${ }^{16} \mathrm{O} /{ }^{18} \mathrm{O}=489$.

${ }^{e}$ Inferred from $\mathrm{H}^{13} \mathrm{CO}^{+}$; assumes ${ }^{12} \mathrm{C} /{ }^{13} \mathrm{C}=60$.

abundances by a factor of 5 . Abundances for the inner core are derived from the $\lambda=3 \mathrm{~mm}$ continuum emission using a $v^{1}$ emissivity; a $v^{2}$ emissivity would decrease the abundances in the inner core by a factor of 12 (see $\S 4.2$ for a detailed dis-

\begin{tabular}{|c|c|c|}
\hline \multirow[b]{2}{*}{ MoleCUle } & \multicolumn{2}{|c|}{$\begin{array}{l}\text { FraCtiONAL ABUNDANCE } \\
\text { ON THE SCALE OF THE }\end{array}$} \\
\hline & Condensation $^{\mathrm{a}}$ & Inner Core ${ }^{b}$ \\
\hline $\mathrm{CO} \ldots \ldots \ldots \ldots$ & $1.4(-5)$ & \\
\hline $\mathrm{CS} \ldots \ldots \ldots \ldots$ & $6.7(-10)$ & $3.3(-9)$ \\
\hline $\mathrm{SiO} \ldots \ldots \ldots$ & $<2.6(-12)$ & $<2.8(-10)$ \\
\hline $\mathrm{CH}_{3} \mathrm{OH} \ldots \ldots$ & $2.6(-9)$ & $\ldots$ \\
\hline HCN ......... & $3.9(-10)$ & $\ldots$ \\
\hline DCN $\ldots \ldots \ldots$ & $8.4(-12)$ & $\ldots$ \\
\hline SO $\ldots \ldots \ldots \ldots$ & $1.6(-10)$ & \\
\hline $\mathrm{HCO}^{+}$. & $4.2(-9)$ & $1.8(-8)$ \\
\hline
\end{tabular}
cussion of the adopted emissivity law).
TABLE 5

Molecular Fractional Abundances from IRAS $05338-0624$

\section{CHEMISTRY}

\subsection{Molecular Abundances in the Condensation}

Using the abundances for TMC-1 as a standard for quiescent gas, IRAS $16293-2422$ as a standard for low-mass starforming regions, the Serpens Northwestern Condensation as a low-/intermediate-mass star-forming region, and Orion-S and the Orion Extended Ridge as high-mass star-forming standards with different amounts of chemical processing, we can examine the chemistry of the IRAS $05338-0624$ region. Looking at the condensation first, Figure 6 displays the abundances in each of these sources; the values for the IRAS $05338-0624$ condensation are given in Table 5. The width of each bar represents a factor of 2 change in the abundance in either direction; this extent roughly covers the uncertainties in the density and temperature. For $\mathrm{CO}$, we see approximately a factor of 10 depletion in IRAS 05338-0624 relative to TMC-1; the value is closest to the abundance in the Serpens condensation, a chemically young, dense condensation.

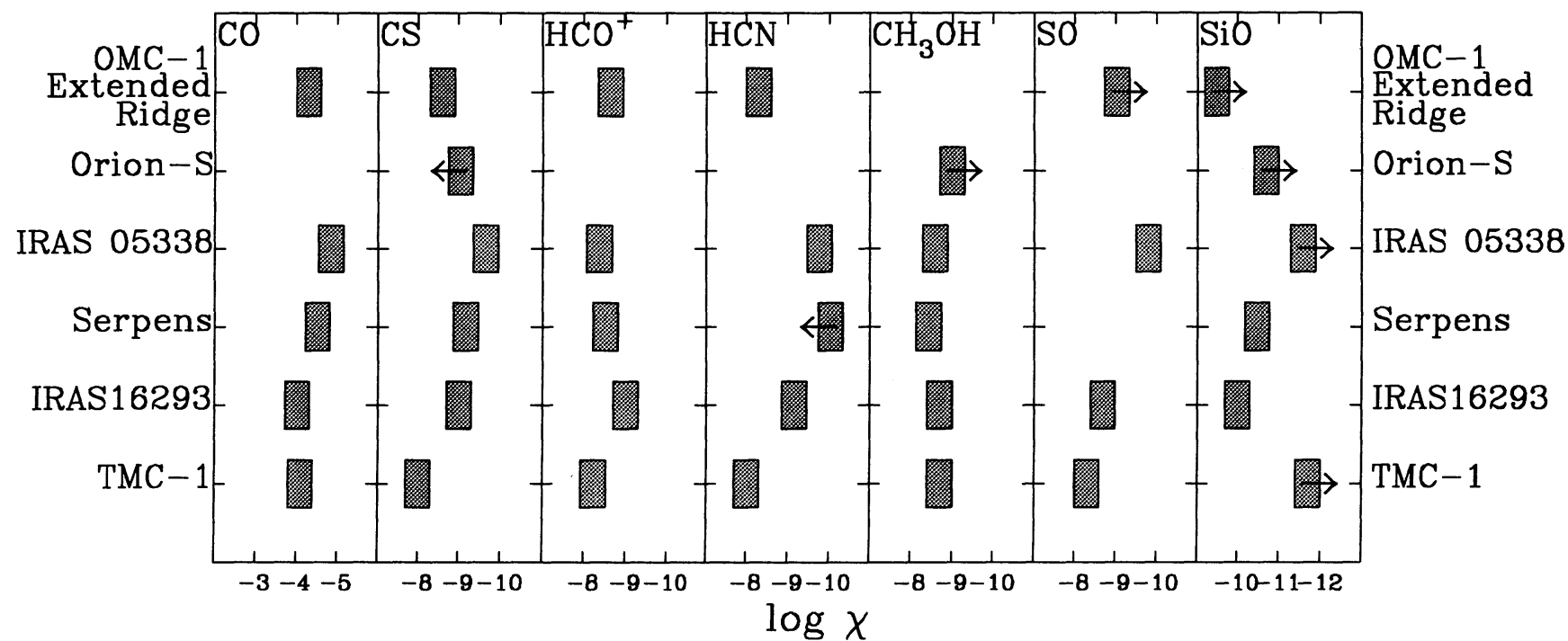

FIG. 6.-Derived abundances for the IRAS $05338-0624$ condensation compared to other star-forming regions and TMC-1. The width of each abundance bar

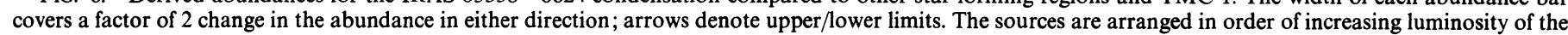

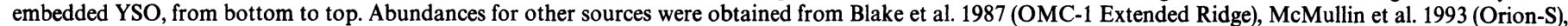
McMullin et al. 1994 (Serpens), Blake et al. 1993 and van Dishoeck et al. 1992 (IRAS 16293-2422), and van Dishoeck et al. 1992 (TMC-1). 
Similarly, the CS abundances in all of the star-forming condensations are approximately a factor of 10 lower than the TMC-1 abundance. SO and HCN show even more dramatic differences, with abundances 30 and $\sim 50$ times lower than in TMC-1, respectively. DCN abundances have not been derived for many of the sources, so DCN was not included in the figure. However, the DCN abundance is also $\sim 30$ times lower than found in TMC-1. The DCN/HCN ratio, which may be an indicator of mantle evaporation, is $\sim 0.02$, a factor of 2000 larger than the standard $\mathrm{D} / \mathrm{H}$ ratio of $10^{-5}$. Such an enhanced ratio is seen toward Orion-KL where it has been postulated that the observed DCN recently sublimated from icy grain mantles that formed at an earlier, colder epoch (Mangum, Plambeck, \& Wootten 1991). The $\mathrm{HCO}^{+}$abundance varies little over the sources surveyed, except in IRAS 16293-2422, which shows a factor of 10 depletion. Overall, for many of these species (CO, CS, HCN, SO), there is a pattern of lower abundances in the star-forming condensations than in the quiescent material as characterized by TMC- 1 or in the gas of OMC-1 Extended Ridge.

$\mathrm{CH}_{3} \mathrm{OH}$ and $\mathrm{SiO}$ show distinctly different trends. The methanol abundance is roughly the same in all of the regions, while the abundance of $\mathrm{SiO}$ shows nearly two orders of magnitude variation. The abundance of $\mathrm{CH}_{3} \mathrm{OH}$ is thought to be enhanced in regions of mild outflow interaction (Millar, Herbst, \& Charnley 1991). Because the interaction is weak, the methanol emission is not necessarily kinematically segregated from the surrounding condensation. As will be shown in the next section, we see evidence for enhanced $\mathrm{CH}_{3} \mathrm{OH}$ abundances in the kinematically isolated shocked gas. Such enhancements may be also occurring in material at condensation velocities resulting in apparent $\mathrm{CH}_{3} \mathrm{OH}$ abundances similar to quiescent material.

As in TMC-1 and the OMC-1 Extended Ridge, the IRAS $05338-0624$ condensation shows no $\mathrm{SiO}$ emission yielding an upper limit to the $\mathrm{SiO}$ abundance which represents a depletion by a factor of greater than 10 from the abundance measured in Serpens and a factor of greater than 35 from that toward IRAS
16293-2422. In IRAS 05338-0624 and Orion-S, the SiO emission peaks at positions offset from the continuum source in regions of suspected shock activity; we will return to this issue in the discussion of the kinematically isolated shocked gas.

Based on these results, we see convincing evidence for gas phase depletions of fundamental gas tracers $(\mathrm{CO}, \mathrm{CS}, \mathrm{HCN}$, $\mathrm{SO})$ on condensation scales.

\subsection{Molecular Abundances in the Inner Core}

To derive abundances for gas in the immediate vicinity of IRAS $05338-0624$, we take the ratio of the molecular column density (within the velocity interval $5-10 \mathrm{~km} \mathrm{~s}^{-1}$ ) with $N_{\mathbf{H}_{2}}$ from the interferometer continuum data. The derived abundances for the inner core are shown in Table 5. Figure 7 illustrates an abundance comparison of IRAS $05338-0624$ with other regions. We see little evidence for gas phase depletions on these scales; since the depletion timescale decreases as the density increases, the denser, inner regions should more readily manifest evidence of adsorption of gas phase molecules onto grain surfaces. The lack of depletions on these scales can be explained in several ways. First, the $\beta=1$ emissivity law used to derive the molecular hydrogen column density biases against depletions (cf. $\S 4.2$ ); if the emissivity law is closer to $\beta=2$, then we are typically seeing depletions of a factor of 10 in the dense gas species ( $\mathrm{CS}$ and $\mathrm{HCO}^{+}$). Second, though we expect depletions to occur rapidly in the dense, inner regions, it is also here that star-forming activity readily deposits its energy. The abundances in this region will change first. As the young star begins interacting with its environment, the larger scale condensation may lag behind in the return of gas-phase tracer molecules as the source evolves.

\subsection{Shock Chemistry}

The direct impact of the outflow from IRAS 05338-0624 on the composition of the surrounding gas can be explored by taking abundance ratios of the different molecular species with respect to a chemically inert species. To accomplish this, we

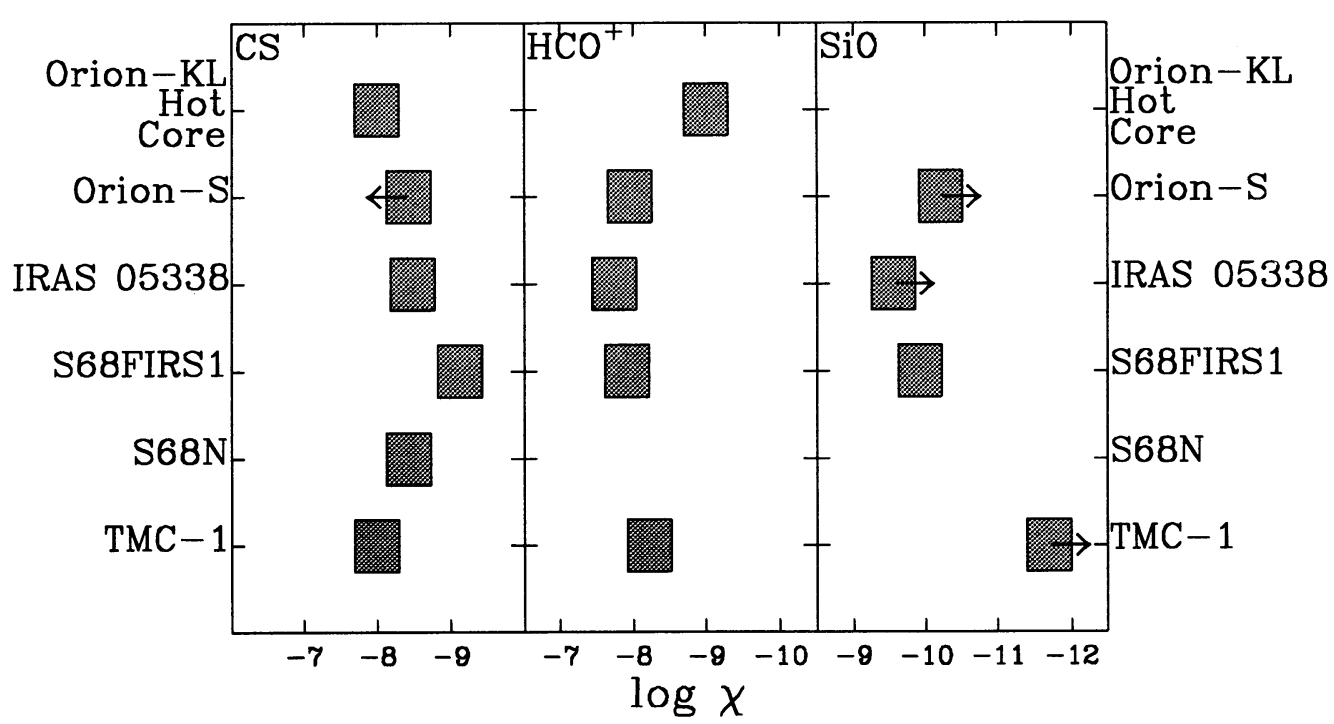

FIG. 7.-Plot of derived abundances for the inner core region around the young stellar object, IRAS 05338-0624, compared to other YSOs and TMC-1. All details are the same as for Fig. 6. Abundances for other sources were obtained from Blake et al. 1987 (Orion-KL Hot Core), McMullin et al. 1993 (Orion-S), McMullin et al. 1994 (S68FIRS1 and S68N), and van Dishoeck et al. 1992 (TMC-1). 
TABLE 6

Relative Fractional Abundances in IRAS 05338-0624

\begin{tabular}{|c|c|c|c|}
\hline \multirow[b]{2}{*}{ MOLECULE } & \multicolumn{3}{|c|}{ Fractional ABUNDANCE } \\
\hline & $\begin{array}{l}\text { Condensation } \\
\text { Ambient Gas }\end{array}$ & $\begin{array}{l}30^{\prime \prime} \text { Resolution } \\
\text { Shocked Gas }\end{array}$ & TMC- $1^{\mathrm{a}}$ \\
\hline $\mathrm{CS} / \mathrm{CO}$ & $1.5(-5)$ & $1.4(-4)$ & $1.3(-4)$ \\
\hline $\mathrm{SiO} / \mathrm{CO}$. & $<1.8(-7)$ & $2.9(-5)$ & $<2.5(-8)$ \\
\hline $\mathrm{CH}_{3} \mathrm{OH} / \mathrm{CO} \ldots \ldots$ & $1.8(-4)$ & $3.4(-3)$ & $2.5(-5)$ \\
\hline $\mathrm{HCN} / \mathrm{CO} \ldots \ldots \ldots$ & $1.2(-5)$ & $1.8(-4)$ & $1.3(-4)$ \\
\hline DCN/CO .......... & $5.8(-7)$ & $\ldots$ & $2.9(-6)$ \\
\hline $\mathrm{SO} / \mathrm{CO} \ldots \ldots$ & $1.1(-5)$ & $2.8(-4)$ & $6.3(-5)$ \\
\hline $\mathrm{HCO}^{+} / \mathrm{CO}$. & $2.9(-4)$ & $\ldots$ & $8.6(-5)$ \\
\hline
\end{tabular}

${ }^{\text {a }}$ From van Dishoeck et al. 1992.

isolate the shocked gas by considering only emission in the velocity interval $10-15 \mathrm{~km} \mathrm{~s}^{-1}$, beyond the systemic velocity of the condensation (see Table 3 for integrated fluxes). We will first consider the observations with $30^{\prime \prime}$ resolution. Using $\mathrm{CO}$ as the "standard" species, we derive the column density ratios given in the second column of Table 6; the corresponding ratios for the ambient gas $\left(5-10 \mathrm{~km} \mathrm{~s}^{-1}\right)$ are given in the first column. The relative abundances of each of the molecules shows a dramatic enhancement in the shocked gas; the enhancements range from a factor of 10 for CS to more than a factor of 160 for $\mathrm{SiO}$. Comparing the ratios to our standard source, TMC-1, we find that the $\mathrm{CS} / \mathrm{CO}$ and $\mathrm{HCN} / \mathrm{CO}$ ratios are the same as in the shocked gas but the other molecules show enhancements ranging from a factor of 4 for SO to more than a factor of 1000 for $\mathrm{SiO}$. These results clearly show that the shocked gas is chemically distinct from the "ambient" material in the condensation.

We can attempt a similar analysis with the $10^{\prime \prime}$ resolution data using CS as the standard dense gas tracer. The ratio of $\mathrm{SiO} / \mathrm{CS}$ column densities at the peak red-wing position shows a factor of greater than 1000 enhancement in the SiO abundance relative to gas in TMC-1 or a factor of greater than 150 enhancement relative to the surrounding condensation.

The observed abundance enhancements in the shocked gas are consistent with models of compositional changes instituted by outflow activity. For example, grain mantle evaporation of sulphur-bearing molecules in warm regions is predicted to drive large SO abundances over time (Charnley 1994). The relatively weak enhancement of SO observed seems to indicate a recent onset for the mantle evaporation or that the temperatures are too low to drive the hot neutral chemistry which produces SO. As noted previously, the $\mathrm{DCN} / \mathrm{HCN}$ ratio also provides evidence for mantle evaporation, and it is likely that the methanol abundance enhancements are also tied to grain mantle evaporation. Recent infrared observations have found large abundances of $\mathrm{CH}_{3} \mathrm{OH}$ in ice mantles (Skinner et al. 1992; Allamandola et al. 1992). Regions of weak interaction between an outflow surface and the surrounding ambient gas could provide sufficient energy to liberate grain mantles without destroying the grains themselves either through sputtering (Jones et al. 1994) or through heating of the grain mantles (Sandford \& Allamandola 1993). Millar et al. (1991) have produced several models that inject amounts of water or methanol into a gas phase chemical model that then evolves according to ion-molecule chemistry. Their methanol-injection models are capable of producing our observed $\mathrm{CH}_{3} \mathrm{OH} / \mathrm{CO}$ abundance ratio with a model using an intermediate injection of methanol gas (between Model 6 and Model 9); the waterinjection models do not reproduce our observed ratio.
In a similar way, the enhanced $\mathrm{SiO}$ abundances are likely to be tied to grain mantle evaporation, and perhaps grain core sputtering. The gas-phase $\mathrm{SiO}$ abundance in molecular clouds is normally severely depressed by gas-phase Si depletion and a proposed activation barrier to formation of $\mathrm{SiO}$ (Zuirys, Friberg, Irvine 1989; Langer \& Glassgold 1990). High-velocity shocks $\left(>100 \mathrm{~km} \mathrm{~s}^{-1}\right)$ are able to return significant amounts of $\mathrm{Si}$ to the gas phase through the destruction of silicate grain cores via sputtering; slower shocks $\left(<100 \mathrm{~km} \mathrm{~s}^{-1}\right)$ are capable of liberating mantle materials which may also include small amounts of Si (Jones et al. 1994; Sandford \& Allamandola 1993). Models of molecule reformation behind dissociative shocks find that $\mathrm{SiO}$ formation proceeds vigorously in warm and Si-rich gas (Neufeld \& Dalgarno 1989). The resulting SiO abundance depends strongly on the shock velocity and preshock gas density; for a preshock density of $10^{5} \mathrm{~cm}^{-3}$ and shock speed in the range from 60 to $100 \mathrm{~km} \mathrm{~s}^{-1}$, the abundance ratio $\mathrm{SiO} / \mathrm{CO}$ varies from $4 \times 10^{-2}$ to $2 \times 10^{-4}$. Hence higher velocity shocks are required to match the observed ratio of $3 \times 10^{-5}$, but such shock velocities produce two orders of magnitude too little $\mathrm{HCN}$ and three orders of magnitude less SO than observed (Neufeld \& Dalgarno 1989). Thus a simple single-velocity shock model does not directly reproduce all of the observed abundances. However, there are caveats to keep in mind. Under the assumption that all of the carbon is in $\mathrm{CO}$, only $0.1 \%$ of the cosmic abundance of $\mathrm{Si}$ is present in the observed $\mathrm{SiO}$, so only a small amount of grain sputtering is needed. Moderate velocity dissociative shocks could return only a fraction of the available Si to the gas phase, resulting in lower $\mathrm{SiO}$ abundances. Nondissociative shocks may also be capable of reproducing the $\mathrm{SiO}$ abundance, as well as the abundances of $\mathrm{CH}_{3} \mathrm{OH}$ and $\mathrm{SO}$, through liberation of mantle materials and small amounts of Si either contained in the grain mantle or sputtered from the grain core. Modeling with closer couplings between shock chemistry and grain destruction is needed to resolve these issues. It is interesting to note that the observed line width of $\mathrm{SiO}\left(\sim 8 \mathrm{~km} \mathrm{~s}^{-1}\right)$ implies much lower shock velocities than are generally required to produce substantial alteration. However, this behavior is consistent with jet-driven models for shocks in which faster shocks are subsequently slowed down by sweeping up ambient material, as seen in NGC 2071 (Chernin \& Masson 1993).

\section{SUMMARY}

We present millimeter wavelength observations of the molecular gas toward IRAS 05338-0624. Based on $\lambda=1 \mathrm{~mm}$ molecular line maps of the dense gas around IRAS $05338-0624$, we derive a characteristic size of $60^{\prime \prime}(0.13 \mathrm{pc})$ for the overall condensation. Isotopic CS observations yield a characteristic density of $2 \times 10^{5} \mathrm{~cm}^{-3}$. Based on the $C^{34} S$ line width and a distance of $450 \mathrm{pc}$, we derive a total virial mass of $40 M_{\odot}$ for the condensation. Based on the interferometer continuum observations and a dust emissivity index of $\beta=1$, we derive a mass for the inner core region of $2 M_{\odot}$, or approximately $5 \%$ of the total mass of the condensation.

A comparison of the derived molecular fractional abundances with quiescent and star-forming regions indicates evidence for gas phase depletions on the scale of the condensation (by factors of 10-50) but no evidence for depletions in the more compact gas associated with the YSO. These results are sensitive to our assumed dust properties; we used a $\beta=2$ emissivity law for the condensation and a $\beta=1$ emissivity law for the inner core. If a $\beta=2$ emissivity is used for the inner core region, then typical depletions of a factor of 10 are seen. 
However, the $\beta=2$ emissivity is not consistent with values derived for the circumstellar regions around other YSOs and yields a mass for the inner core which is approximately the same as that derived for the overall condensation based on continuum observations; the extended emission distributions I. of dense gas tracers and thermal dust as seen in single-dish observations does not support this. We suggest that the absence of depletions in the inner core may be due to the evaporation of grain mantles in response to energy inputs from the YSO; the condensation may lag behind in this process.

Outflow sensitive molecular species ( $\mathrm{SO}, \mathrm{CH}_{3} \mathrm{OH}$, and $\mathrm{SiO}$ ) show pronounced wing emission at a position $8^{\prime \prime}$ south and $14^{\prime \prime}$ west of the IRAS source; interferometer observations show this to be a region of interaction with a bipolar outflow oriented at an angle of $45^{\circ}$. By kinematically isolating the outflowing gas to velocities well beyond the $V_{\mathrm{LSR}}$ of the cloud core and measuring abundances relative to $\mathrm{CO}$, we find relative abundances of $\mathrm{SO}, \mathrm{CH}_{3} \mathrm{OH}$, and $\mathrm{SiO}$ in the shocked gas are elevated by factors of $4-1000$ relative to TMC-1. The relative abundance enhancements are difficult to explain with existing single-velocity shock models, but may be consistent with a nondissociative shock model in which some refractory materials are released along with the evaporating mantles or with a moderate velocity dissociative shock in which only a moderate sputtering of $\mathrm{Si}$ in grain cores or mantles occurs. Additionally, a shock that impacts on surrounding material over a range of speeds may also explain the measured abundances.

We would like to thank Jocelyn Keene, Tamara Helfer, and John Howe for help with observations. We thank Phil Jewell and the telescope operators of the NRAO 12 meter for their kind assistance as well as Rick Forster, Lanie Dickel and the BIMA observers. We are grateful to our anonymous referee, Gary Fuller, and Ken Tatematsu for helpful comments on this work. This work was sponsored by National Science Foundation grant AST 9314847 and by NASA grant NAGW-3066. G. A. B. acknowledges support from the David \& Lucille Packard and Alfred P. Sloan Foundations, and NASA grant NAGW-2297.
Allamandola, L. G., Sandford, S. A., Tielens, A. G. G. M., \& Herbst, T. M. 1992, ApJ, 399, 134

Beckwith, S. V. W., \& Sargent, A. I. 1991, AJ, 381, 250

Blake, G. A., Sutton, E. C., Masson, C. R., \& Phillips, T. G. 1987, ApJ, 315, 621

Blake, G. A., van Dishoeck, E. F., Jansen, D. J., Groesbeck, T., \& Mundy, L. G. 1994, ApJ, 428, 680

Blake, G. A., van Dishoeck, E. F., \& Sargent, A. I. 1992, ApJ, 391 , L99

Charnley, S. B. 1994, ApJ, submitted

Chen, H., Fukui, Y., \& Yang, J. 1992, ApJ, 398, 544

Chernin, L., \& Masson, C. 1993, ApJ, 403, L21

Chini, R., Krugel, E., \& Kreysa, E. 1986, A\&A, 167, 315

Draine, B. T. 1990, in The Interstellar Medium in Galaxies, ed. H. A. Thronson \& J. M. Shull (Dordrecht: Kluwer), 483

Draine, B. T., \& Lee, H. M. 1984, ApJ, 285, 89

Fukui, Y., Takaba, H., Iwata, T., \& Mizuno, A. 1988, A\&A, 166, 176

Glassgold, A. E., Mamon, G. A., \& Huggins, P. J. 1989, ApJ, 336, L29

Goldsmith, P. F., Margulis, M., Snell, R. L., \& Fukui, Y. 1992, ApJ, 385, 522

Harju,, J., Walmsley, C. M., \& Wouterloot, J. G. A. 1991, A\&A, 245, 643

Hildebrand, R. H. 1983, QJRAS, 24, 267

Jewell, P. R. 1988, NRAO 12 meter Telescope Receiver and Calibration Status

Jones, A. P., Tielens, A. G. G. M., Hollenbach, D. J., \& McKee, C. F. 1994, ApJ, 433, 797

Lada, C. 1985, ARA\&A, 23, 267

Langer, W. D., \& Glassgold, A. E. 1990, ApJ, 352, 121

Mangum, J. G., Plambeck, R. L., \& Wootten, A. 1991, ApJ, 369, 169

Mauersberger, R., Wilson, T. L., Mezger, P. G., Gaume, R., \& Johnston, K. J. 1992, A\&A, 256, 640

McMullin, J. P., Mundy, L. G., \& Blake, G. A. 1993, ApJ, 405, 599

McMullin, J. P., Mundy, L. G., Wilking, B. A., Hezel, T., \& Blake, G. A. 1994, ApJ, 424, 222

\section{EFERENCES}

Miller, T. J., Herbst, E., \& Charnley, S. B. 1991, ApJ, 369, 147

Miyake, K., \& Nakagawa, Y. 1993, Icarus, 106, 20

Neufeld, D. A., \& Dalgarno, A. 1989, ApJ, 340, 869

Plambeck, R. L., \& Wright, M. C. H. 1987, in Molecular Clouds in the Milky Way and External Galaxies, ed. R. L. Dickman, R. L. Snell, \& J. S. Young (New York: Springer), 182

Rawlings, J. M., Hartquist, T., Menten, K., \& Williams, D. A. 1992, MNRAS, 255,471

Rudolph, A., \& Welch, W. J. 1988, ApJ, 326, L31

Sandford, S. A., \& Allamandola, L. J. 1993, ApJ, 417, 815

Schwartz, P. R. 1982, ApJ, 252, 589

Shu, F., Najita, J., Galli, D., \& Ostriker, E. 1993, in Protostars and Planets III, ed. E. H. Levy \& J. I. Lunine (Tucson: Univ. Arizona Press), 3

Skinner, C. J., Tielens, A. G. G. M., Barlow, M. J., \& Justtanont, K. 1992, ApJ, 399, L79

Strom, K. M., Margulis, M., \& Strom, S. E. 1989, ApJ, 346, L33

Takaba, H., Fukui, Y., Fujimoto, Y., Sugitani, K., Ogawa, H., \& Kawabata, K. 1986, A\&A, 166, 276

Van Dishoeck, E. F., Blake, G. A., Draine, B. T., \& Lunine, J. I. 1992, in Protostars and Planets III, ed. E. H. Levy, J. I. Lunine, \& M. S. Matthews (Tucson: Univ. Arizona Press), 163-241

Walker, C. K., Adams, F. C., \& Lada, C. J. 1990, ApJ, 349, 515

Weintraub, D. A., Sandell, G., \& Duncan, W. D. 1991, ApJ, 382, 270

Whittet, D. C. B. 1988, in Dust in the Universe, ed. M. E. Bailey \& D. A Williams (Cambridge: Cambridge Univ. Press), 25

Wilking, B. A., Blackwell, J. H., \& Mundy, L. G. 1990, AJ, 100, 758

Wilking, B. A., Mundy, L. G., Blackwell, J. H., \& Howe, J. E. 1989, ApJ, 345, 257

Woody, D. P., et al. 1989, ApJ, 337, L41

Ziurys, L. M., Friberg, P., \& Irvine, W. M. 1989, ApJ, 343, 201 